\title{
Aeroacoustic noise reduction by application of end plates on wall-mounted finite airfoils
}

\author{
Erik Schneehagen ${ }^{1} \cdot$ Thomas F. Geyer $^{2} \cdot$ Ennes Sarradj $^{1} \cdot$ Danielle J. Moreau ${ }^{3}$
}

Received: 16 November 2020 / Revised: 1 April 2021 / Accepted: 6 April 2021 / Published online: 24 April 2021

(c) The Author(s) 2021

\begin{abstract}
One known method to reduce vortex shedding from the tip of a blade is the use of end plates or winglets. Although the aerodynamic impact of such end plates has been investigated in the past, no studies exist on the effect of such end plates on the far-field noise. The aeroacoustic noise reduction of three different end-plate geometries is experimentally investigated. The end plates are applied to the free end of a wall-mounted symmetric NACA 0012 airfoil and a cambered NACA 4412 airfoil with an aspect ratio of 2 and natural boundary layer transition. Microphone array measurements are taken in the aeroacoustic open-jet wind tunnel at BTU Cottbus-Senftenberg for chord-based Reynolds numbers between 75,000 and 225,000 and angles of attack from $0^{\circ}$ to $30^{\circ}$. The obtained acoustic spectra show a broad frequency hump for the airfoil base configurations at higher angles of attack that is attributed to tip noise. Hot-wire measurements taken for one configuration show that the application of an end plate diffuses the vorticity at the tip. The aeroacoustic noise contribution of the tip can be reduced when the endplates are applied. This reduction is most effective for higher angles of attack, when the tip vortex is the dominant sound source.
\end{abstract}

\section{Graphic abstract}
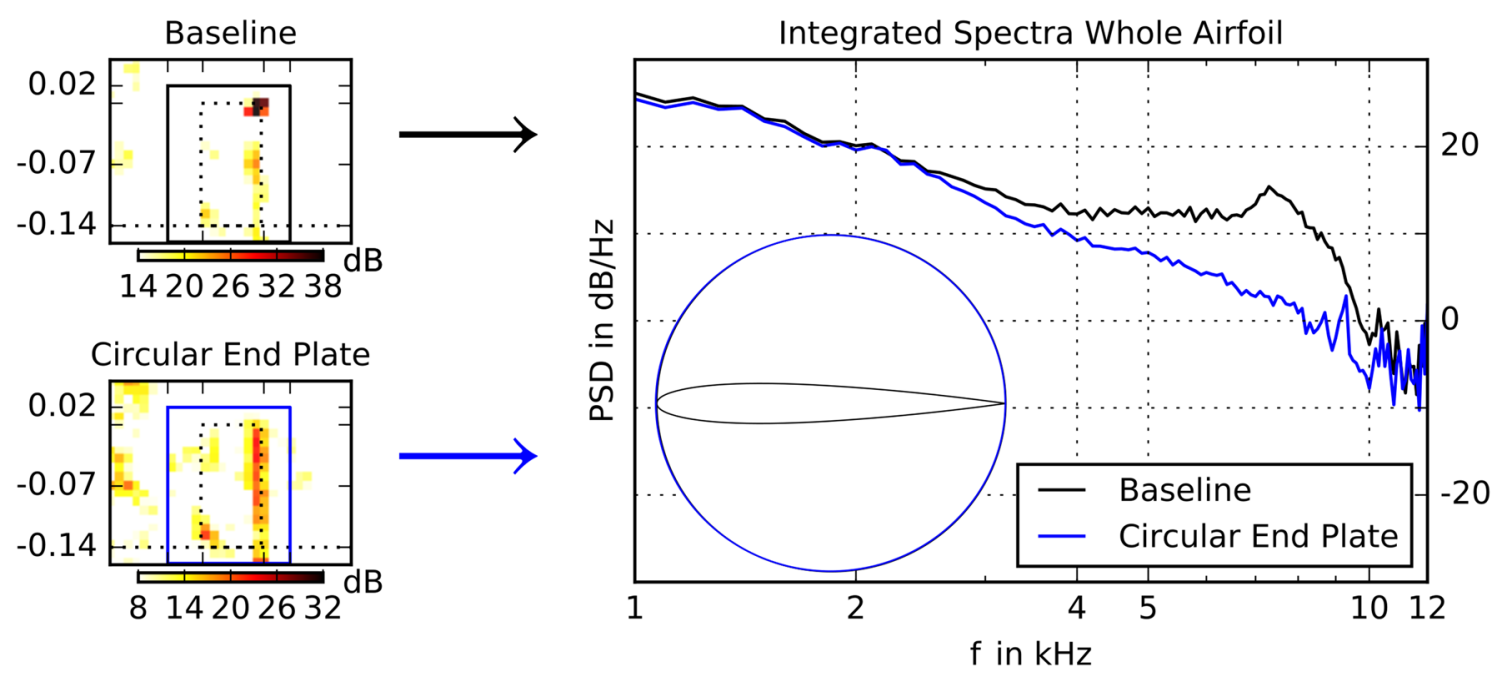

Erik Schneehagen

erik.w.schneehagen@tu-berlin.de

Extended author information available on the last page of the article

\section{Introduction}

For all lifting airfoils in a flow, regions of high and low pressure are created by the flow over the pressure side surface and the suction side surface, respectively. If the airfoil is finite and hence has a free end exposed to the flow, the resulting pressure gradient between both sides at this end 
can force the flow around the tip side edge. The tip vortex generated by this spanwise flow is not only responsible for induced drag but can also be a significant source of broadband flow-induced noise. Guo (2011) developed a semi-empirical model for flap side edge noise in which two noise generation mechanisms are identified: the flow separation near the sharp corners of the flap and the interaction of large-scale vortex structures with the sharp corners of the flap. For low Mach number flows, the resulting pressure fluctuations on the surface are efficient noise radiators. Thereby, the magnitude of the sound radiation strongly depends on the strength of the vorticity, which is created by the sweep of the flow around the edge as well as on the distance of the vortex from the edge (Hardin 1980). Studies on airfoil tip noise by Moreau et al. (2016), Moreau and Doolan (2016) demonstrated that the dominant tip noise contribution manifests itself as a broad peak or hump in the noise spectrum. The broad tip noise peak increases in amplitude as the angle of attack is increased and, to a lesser extent, when flow speed is increased.

For aircraft airframe components including the main wing and flap side edge, it is crucial to not only reduce noise to meet regulations but also to increase the aerodynamic efficiency. Especially for airfoils used in rotating applications, like wind turbine blades, the noise contribution from the tip is very important. This is due to the fact that the flow velocity along the span takes maximum values in this region (George et al. 1980) and that the resulting tonal noise occurs at frequency ranges where it can be perceived as particularly annoying.

While the physics of this phenomenon is quite well understood and corresponding scaling models exist (George et al. 1980; Brooks and Marcolini 1986; Moreau et al. 2016), measures for preventing the tip vortex formation are not extensively studied. In general, three different, passive measures exist: one is the introduction of porous material at the tip in order to reduce the pressure jump between suction and pressure side and therefore reduce the maximum strength of the vorticity (Revell et al. 1997; Angland et al. 2009). Another measure is to modify the geometry of the tip region with the aim to minimize the interaction between the vortices and the side edge (Kinzie et al. 2013) and the third is the application of winglet-type fences to diffuse the vorticity at the edge by creating a physical obstacle from top to bottom (Slooff et al. 2002; Zaman et al. 2017). The latter is also commonly used to reduce airfoil drag in flight. While the flow field and vortex generation is investigated in detail in these studies, the aeroacoustic effect of these measures is considered very little or not at all.

This paper aims to study the effect of end plates applied to the free end of a wall-mounted finite airfoil on the resulting noise generation. For this purpose, two airfoil shapes are considered, which are widely used in studies on airfoil aerodynamics and aeroacoustics: the symmetric NACA 0012 airfoil and the cambered NACA 4412 airfoil. They are often used in low-speed applications such as drone/UAV propeller blades at low-to-mid Reynolds numbers. Due to the difference in camber and the resulting differences in surface curvature, these airfoils are known to show quite different behaviour regarding flow separation and transition (Zhang et al. 2015). The symmetrical NACA 0012 airfoil develops a stronger adverse pressure gradient, which leads to the fact that flow detaches at a position further upstream than for the cambered NACA 4412 airfoil. To illustrate the differences, Fig. 1 shows the pressure coefficient calculated for a NACA 0012 airfoil and a NACA 4412 airfoil at an angle of attack of $15^{\circ}$ using XFOIL (Drela 1989).

The investigated end-plate geometries are taken from an early study on the aerodynamic characteristics of an unswept NACA $64{ }_{1}$ A412 airfoil at a Reynolds number (based on
Fig. 1 Pressure coefficient of a 2D NACA 0012 airfoil versus that of a NACA 4412 airfoil [calculated for inviscid flow at $R e=125,000$ and $M=0.0787$ using XFOIL (Drela 1989)]

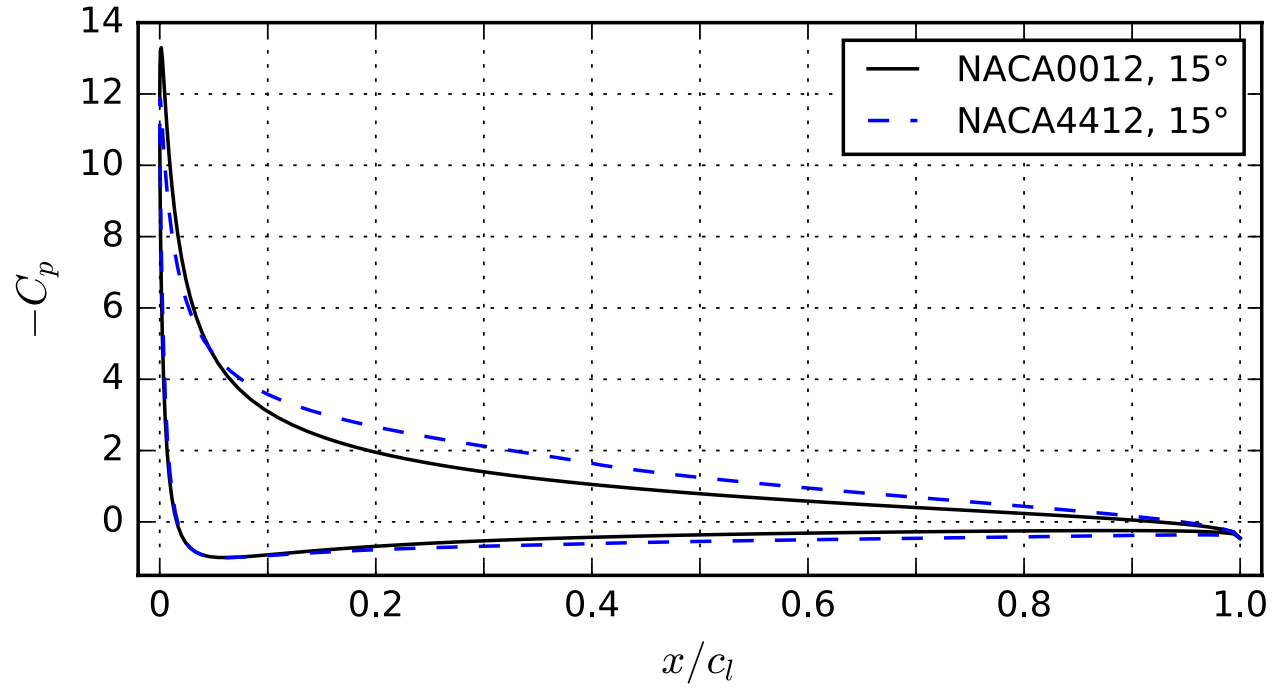


airfoil chord length) of $10^{6}$ with multiple different end-plate designs (Riley 1951). Three of these end-plate geometries which are easy to manufacture and show the most beneficial aerodynamic characteristics are considered in the current paper. To show the favourable aerodynamics of these end plates, results from the original study by Riley (1951) are displayed in Fig. 2. In Fig. 2(a), it can be seen that the lift coefficient $C_{L}$ is larger for all angles of attack when end plates are applied to both sides of the airfoil. It is possible that the end plates effectively change the shape of the wing or slightly increase the effective span. Figure 2(b) then shows that the lift-drag ratio is higher with end plates for large lift coefficients. The width of the range where the liftdrag ratio takes maximum values also increases, although the maximum value is higher for the base configuration without end plate.
Apart from these characteristics, the effect of an applied end plate on the flow around an airfoil was examined qualitatively by performing basic flow visualization experiments in the UNSW wind tunnel (Doolan et al. 2018). The results are shown exemplary in Fig. 3. For these photographs, taken from the suction side, smoke was introduced to the flow upstream of a NACA 4412 airfoil at $15^{\circ}$ angle of attack and $R e=50,000$. The photographs show clear paths of the flow as white smoke streamlines. The top view of the baseline configuration (Fig. 3a) shows an absence of smoke in the wake of the airfoil close to the free end. It can be assumed that this is caused by the tip vortex. For the case when the circular end plate is applied to the free end of the airfoil (Fig. 3b), no such absence of smoke can be seen, as the smoke seems to be distributed more homogeneously in the wake. Thus, it appears that the end plate suppresses the vortex formation at the tip. (a)

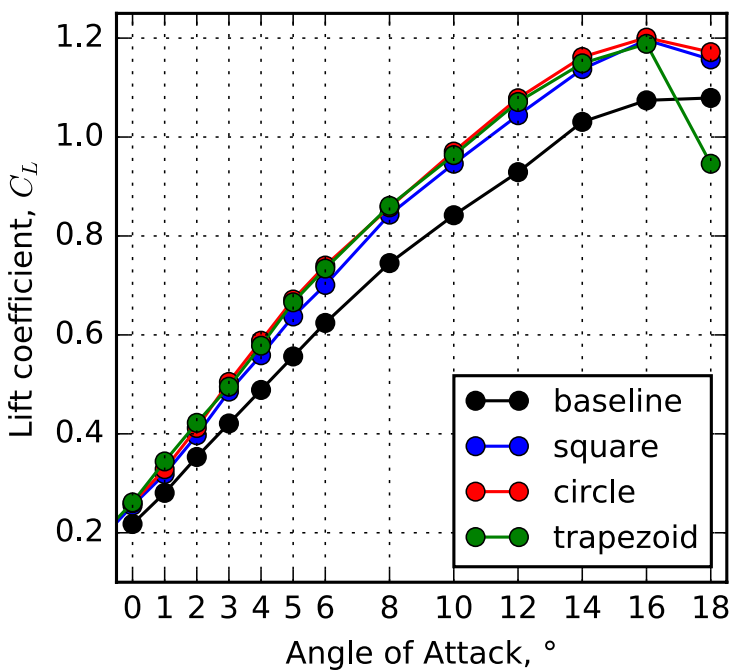

(b)

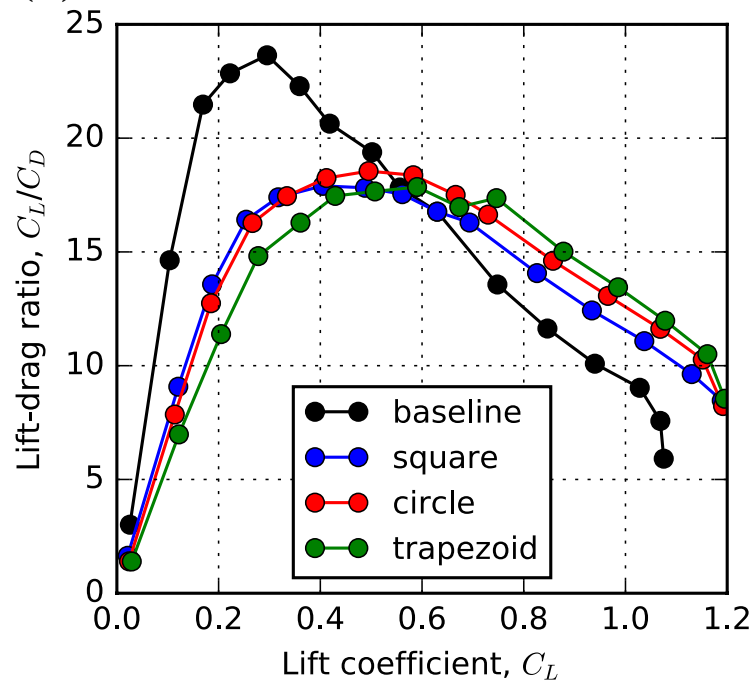

Fig. 2 Aerodynamic characteristics of single NACA $64{ }_{1}$ A412 (baseline) and in combination with various end plates, data replotted from Riley (1951)

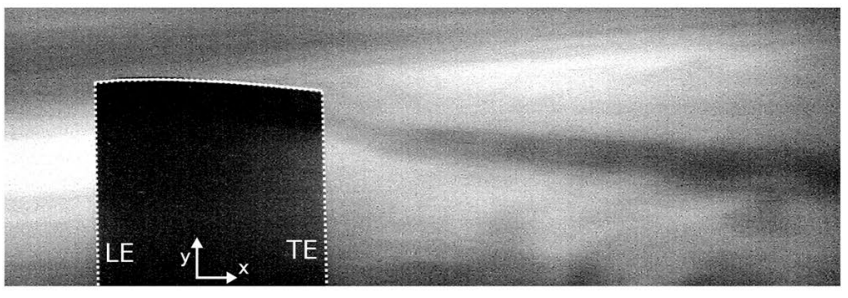

(a) Baseline (flat tip, no end plate)

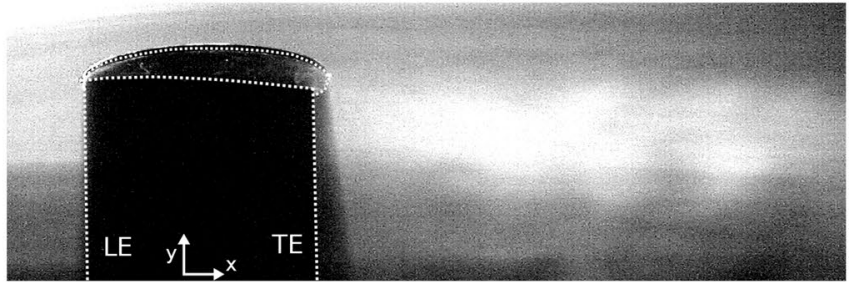

(b) Circular endplate

Fig. 3 Flow visualization performed for a NACA 4412 airfoil with/without end plate, $R e=50,000,15^{\circ}$ angle of attack, top view suction side with airfoil edges as white dotted lines, flow from left to right 
For this modification of the flow field, the current study aims to add an aeroacoustic point of view and present the noise reduction potential of these end plates for flow configurations where tip noise is dominant. Therefore, the noise reduction effect is experimentally investigated in an aeroacoustic wind tunnel for symmetric (NACA 0012) and cambered (NACA 4412) airfoils with natural boundary layer transition. The dataset presented in this paper includes microphone array measurements for chord-based Reynolds numbers from 75,000 to 225,000 in increments of 25,000 . The angle of attack is varied from $0^{\circ}$ to $25^{\circ}$ for the NACA 0012 airfoil and from $0^{\circ}$ to $30^{\circ}$ for the NACA 4412 airfoil in $2.5^{\circ}$-steps. Additionally, hot-wire measurements were taken for one configuration in order to obtain more details on the flow field and to relate this to the noise reduction mechanism.

The paper is structured as follows: In Sect. 2, the experimental set-up and methods are described. Section 3 presents acoustic spectra and sound maps from microphone array measurements and flow velocity fields from hot-wire measurements in the wake of the airfoil tip. Finally, Section 4 gives concluding remarks.

\section{Experimental method}

\subsection{Airfoil models}

The two airfoil profiles NACA 0012 and NACA 4412 have a theoretical chord length $C$ of $70 \mathrm{~mm}$ and span $L$ of $140 \mathrm{~mm}$ resulting in the aspect ratio $L / C=2$. The actual chord length is $67 \mathrm{~mm}$ due to a truncated rounded trailing edge with curvature diameter of $1.0 \mathrm{~mm}$. These airfoils follow the NACA four-digit series and have $0 \%$ and $4 \%$ camber at $40 \%$ chord, respectively. For some selected configurations, forced boundary layer transition was achieved by using 60 -degree zigzag trip tape (manufactured by Glasfaser Flugzeugservice) with $0.4 \mathrm{~mm}$ thickness and $6 \mathrm{~mm}$ point-to-point distance on both sides of the airfoil at $10 \%$ chord. The three analysed end-plate geometries (Riley 1951) are shown in Fig. 4. All end plates have the theoretical chord length of $70 \mathrm{~mm}$. The height of the square plate (a) is 0.5 chord lengths, the radius of the circular plate (b) is one chord length, and the height of the trapezoidal end plate is 0.1 chord lengths at the leading edge and 1.2 chord lengths at the trailing edge. The end plates were attached to the airfoils with double-faced adhesive tape. The end plates were manufactured out of 2-mm-thin plastic (polyurethane) plates. The edges are straight and untreated.

\subsection{Wind tunnel}

The measurements were taken in the aeroacoustic open jet wind tunnel at BTU Cottbus-Senftenberg (Sarradj et al. 2009). The experimental set-up can be seen in Fig. 5a. The two airfoil profiles, NACA 0012 and NACA 4412, were attached to an acrylic glass plate, which was fixed on the rectangular nozzle exit (green), which has the width of $280 \mathrm{~mm}$ and height of $230 \mathrm{~mm}$. At the nozzle exit, the flow is characterized by an even profile and a very low turbulence intensity in the order of $0.2 \%$ (Geyer et al. 2015). The thickness of the boundary layer on the side plate at the position of the airfoil leading edge is in the order of $5 \mathrm{~mm}$ to $10 \mathrm{~mm}$ at flow speeds ranging from 10 to $50 \mathrm{~m} / \mathrm{s}$ (Moreau et al. 2017). Due to the small size of the airfoils compared to the nozzle exit area, no blockage correction was performed. The microphone array was mounted on the ceiling of the test section, and all other surrounding walls were covered with sound-absorbing material, which provides an anechoic environment for frequencies above $125 \mathrm{~Hz}$.
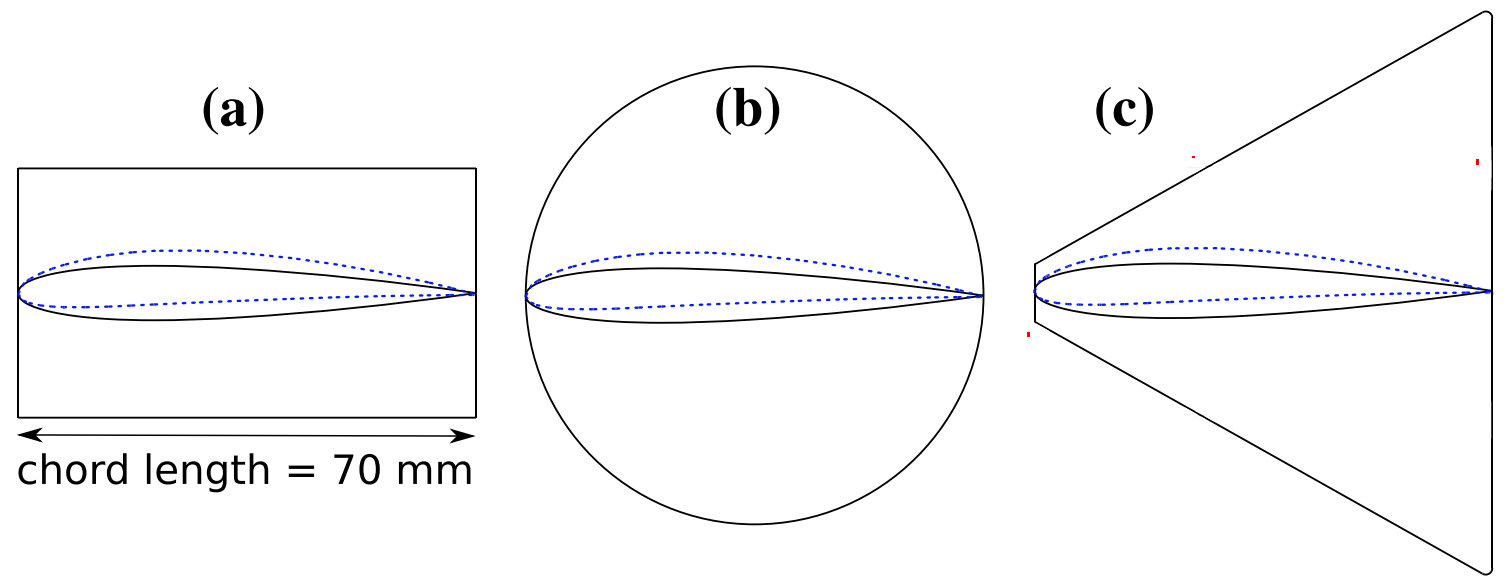

Fig. 4 End-plate geometries with NACA 0012 (solid, black line) and NACA4412 (dashed, blue line) outline: a square (height 0.5 chord lengths), b circle (radius 1.0 chord length), c trapezoid (height 0.1 to 1.2 chord lengths) 


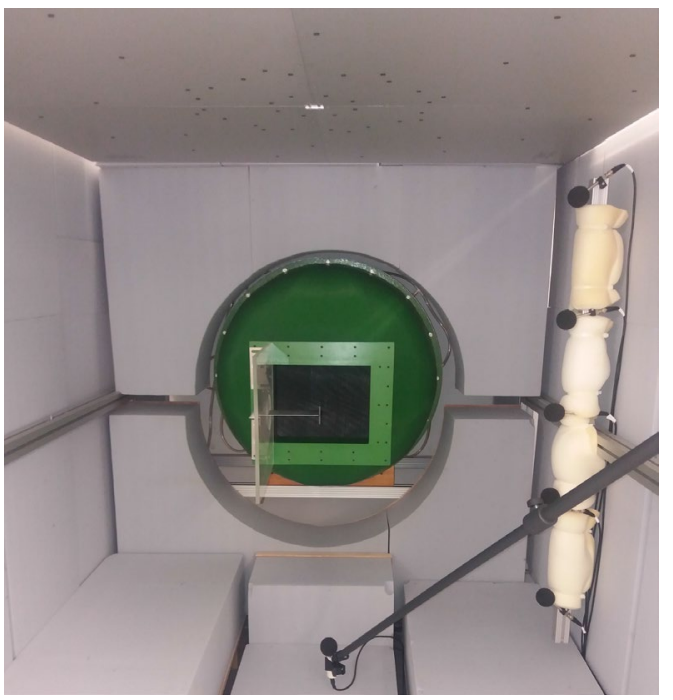

(a) Aeroacoustic wind tunnel at BTU Cottbus-Senftenberg

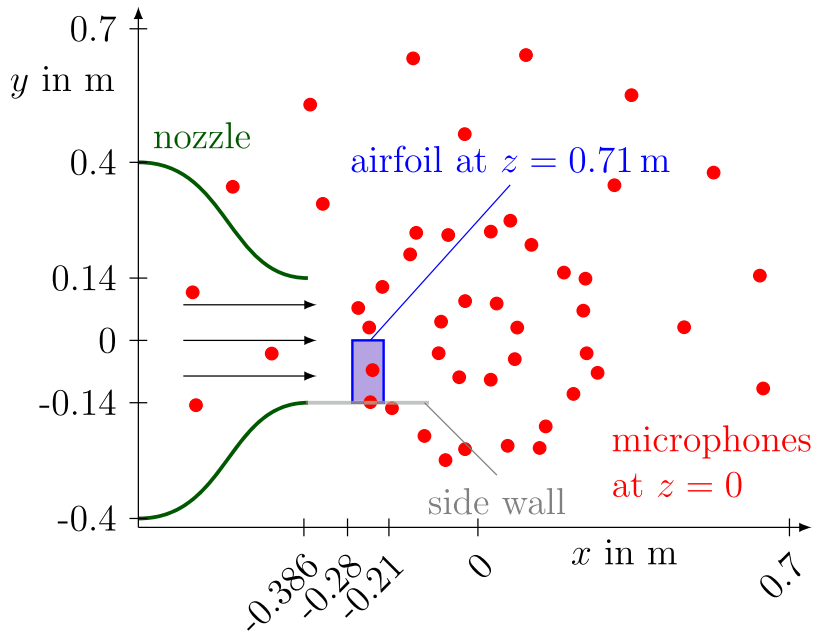

(b) schematic top view through the ceiling

Fig. 5 Acoustical measurement set-up in aeroacoustic wind tunnel, nozzle (green), microphone array in ceiling and microphone positions (red)

The angle of attack was adjusted with an electronic angle meter, which was placed on a ledge parallel to the airfoil chord line. It has to be noted that for a finite length airfoil with flow over the tip, the effective angle of attack changes along the span, which will also affect the lift coefficient. For the configurations without end plates, the angle of attack distribution can be calculated using the procedure reported by Awasthi et al. (2018). No method exists for the calculation of an effective angle of attack that considers the presence of end plates. Hence, it was decided to perform all far-field noise comparisons at the same geometric angles of attack and not at the same lifting conditions. However, it should be kept in mind that the airfoil generates more lift at the same geometric angle of attack when end plates are applied (see Fig. 2a) and also the lift-drag ratio is altered (Fig. 2b). Although the original study by Riley (1951) was done with a different airfoil profile and two free ends, the general trend of the aerodynamic characteristics still provides some insight.

\subsection{Acoustic measurement set-up and data processing}

The acoustic measurements were taken using a planar microphone array consisting of 47 1/4-inch Panasonic microphone capsules (WM-61A), which were flush-mounted on the rigid ceiling. The microphone distribution together with the coordinate system is shown in Fig. 5b. The axis origin matches the array centre, while $x$ - and $y$ - were the streamwise and spanwise axis, respectively. The distance to the airfoil trailing edge at $0^{\circ}$ angle of attack was $710 \mathrm{~mm}$.
All pressure signals were recorded over a duration of 40 seconds with a National Instruments 24-bit multichannel system, (including PXI-4472 cards in a NI PXI-1044 chassis). The sampling frequency was $51.2 \mathrm{kHz}$.

The Acoular 19.02 framework (Sarradj and Herold 2017) together with the CLEAN-SC beamforming algorithm (Sijtsma 2007) was used to locate and evaluate the sound sources in a $40 \mathrm{~cm}$ x $40 \mathrm{~cm}$ focus grid with a grid resolution of $1 \mathrm{~cm}$. This algorithm was chosen due to its fast and stable performance, especially for aeroacoustic testing (Herold and Sarradj 2017; Merino-Martínez et al. 2019; Bahr et al. 2017; Sarradj et al. 2017). Hanning window with the block size of 4096 samples and 50\% overlap was applied for calculating the cross-spectral matrices resulting in the frequency resolution of $12.5 \mathrm{~Hz}$. Additionally, the downstream convection of the sound waves by the flow was accounted for by using a ray-tracing approach together with an analytical approximation of the flow field of a slot jet (Albertson et al. 1950) over the nozzle width. The main diagonal of the averaged cross-spectral matrix was removed to avoid contamination of the signals by incoherent noise (Hald 2017). A value of $6 \mathrm{~dB}$ was subtracted from the sound pressure level spectra to account for reflections at the rigid microphone array plate.

For all the baseline cases (no end plate attached) at angles of attack from $0^{\circ}$ to $12.5^{\circ}$ the dataset from Zhang et al. (2020) was used. These measurements were taken with the same measurement set-up as described here.

The beamforming results were used to quantitatively analyse the sound pressure at the centroid of the array microphone positions $(0,0.07,0)$, which is not exactly at the array centre due to the non-symmetric microphone distribution. In 
order to investigate the locations of dominant sound sources, the source region was subdivided into three different areas from which the contribution to the overall sound pressure level was evaluated. In Fig. 6, these integration sectors are shown.

The whole integration area expands to half a chord length upstream the leading edge and downstream the trailing edge. In the spanwise direction, it expands a third chord length further than junction and tip. The tip integration area is onefourth of the whole span, and the integration sectors of leading and trailing edge are three quarters of span, respectively, and end at half chord. The integrated sound pressure levels from these areas were calculated from $1 \mathrm{kHz}$ to $20 \mathrm{kHz}$. The frequency range is chosen so that the Helmholtz number of the array aperture and the lowest frequency is not lower than 4 to ensure an accurate performance of the CLEAN-SC algorithm (Herold and Sarradj 2017).

\subsection{Hot-wire measurement set-up}

In order to investigate the flow structures in the wake of the tip, constant temperature anemometry (CTA) measurements were taken on the NACA 0012 airfoil for an angle of attack of $15^{\circ}$ and a Reynolds number of 125,000 for the base configuration and the square and circular end plate. A Dantec miniature $\mathrm{X}$-wire probe 55P64 was used to measure the velocity fluctuations in both the streamwise direction $x$ and in the direction $z$ perpendicular to the flow and the span/trailing edge. Additionally, a Dantec 90P10 temperature probe was used for temperature correction during the measurements. The velocity calibration of the hot-wire probe was performed at twelve logarithmically spaced calibration points against a vane anemometer with an accuracy of $\pm 0.2 \mathrm{~m} / \mathrm{s} / \pm 2 \%$ and a measurement range of $0.4 \mathrm{~m} / \mathrm{s} \ldots$ $40 \mathrm{~m} / \mathrm{s}$ using a fourth-order polynomial curve fit. The hotwire measurement system contains an internal low-pass filter with a cut-off frequency of $10 \mathrm{kHz}$, and both probes were connected to a 24 Bit National Instruments digital signal acquisition module with a sampling frequency of $25.6 \mathrm{kHz}$. The probe was positioned using a three-dimensional traverse system with a minimum step size of $0.1 \mathrm{~mm}$. The traverse speed was set low enough to avoid any vibrations of the probe.

The flow velocity time signal was measured for 8 seconds at discrete points in the $y z$-plane, 0.1 chord lengths downstream of the trailing edge. The plane dimensions stretched spanwise from $5 \mathrm{~mm}$ over the tip to $22 \mathrm{~mm}$ spanwise of the airfoil and in the other dimension from $55 \mathrm{~mm}$ above the trailing edge to $33 \mathrm{~mm}$ beneath it. The resolution was $1 \mathrm{~mm}$ in the direct vicinity of the trailing edge and $2 \mathrm{~mm}$ elsewhere. This resulted in a total of 1196 measurement points per configuration. At each point, the mean velocity components $U$ (in streamwise direction $x$ ) and $W$ (in vertical direction $z)$ as well as the total turbulence intensity $\sqrt{\left(u_{\mathrm{rms}}^{2}+w_{\mathrm{rms}}^{2}\right)} / U_{0}$ were calculated, with $u_{\mathrm{rms}}$ and $w_{\mathrm{rms}}$ being the root-mean-square values of the velocity fluctuations in streamwise and vertical (upwash) directions, respectively, and $U_{0}$ being the outer flow speed.
Fig. 6 Different integration sectors for sound pressure calculation out of beamforming results, the airfoil (black) is seen from top view, LE (leading edge), TE (trailing edge)

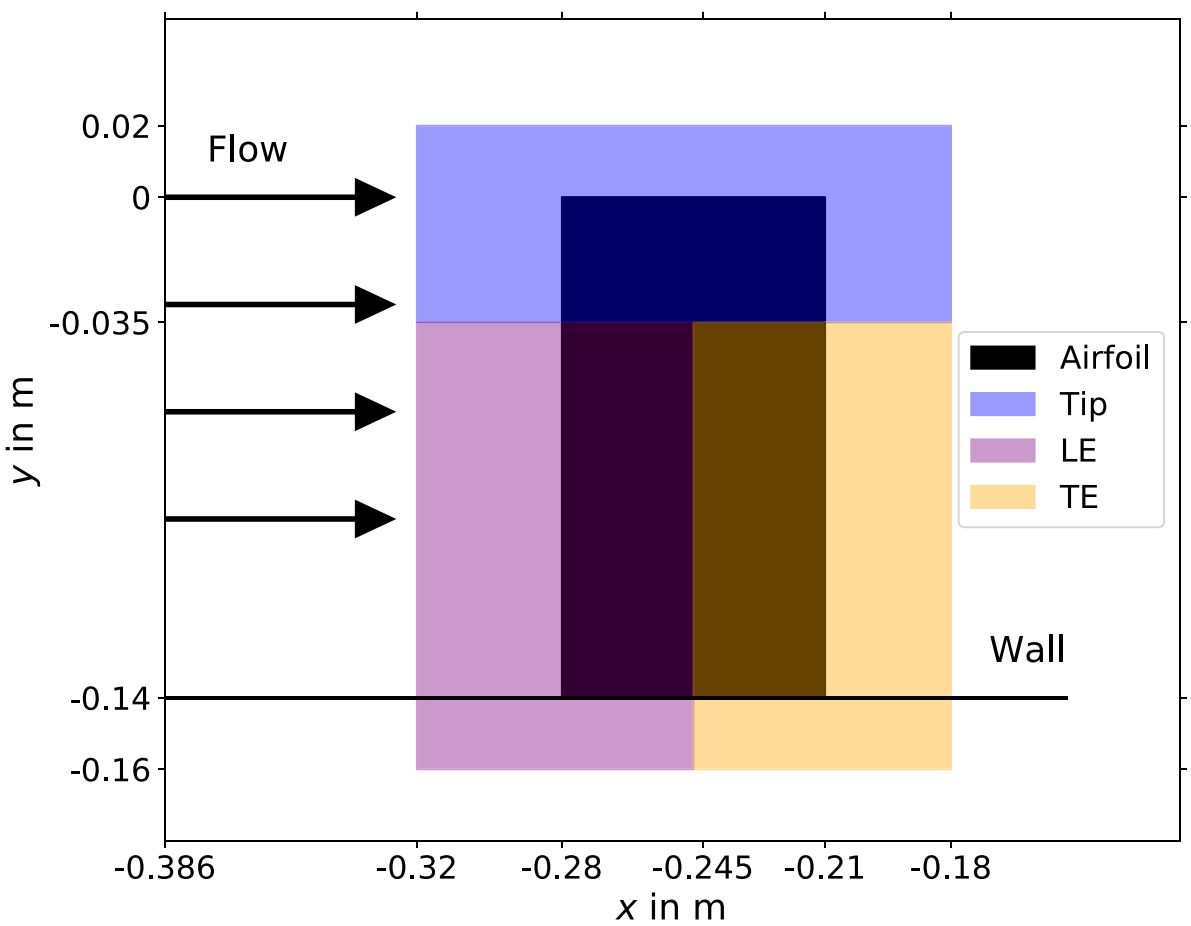




\section{Experimental results}

\subsection{Beamforming results-NACA 0012}

First the procedure of evaluating the noise reduction effect is explained by means of an example. Figure 7 shows the sound maps for one frequency band of the far-field noise measured for the NACA 0012 airfoil at $15^{\circ}$ angle of attack at a Reynolds number of 125,000 for the baseline configuration and the circular end plate attached. For every frequency, the sound pressure level (SPL) of the sources is integrated within the shown area around the whole airfoil (Tip + LE + TE, Fig. 6). This results in the spectra shown for both configurations and is also done for the empty wind tunnel at the same flow speed. The overall sound pressure level (OASPL) is calculated for all frequencies between $1 \mathrm{kHz}$ and $20 \mathrm{kHz}$ where the signal-to-noise ratio to the empty wind tunnel is above $6 \mathrm{~dB}$ for both configurations. The total noise reduction achieved by applying the end plate then corresponds to the difference of these two overall sound pressure levels and amounts to $1.3 \mathrm{~dB}$ in this example.

This procedure is done for all three end-plate configurations of the NACA 0012 airfoil. For the square and the circle end plates, the angle of attack is varied from $0^{\circ}$ to $25^{\circ}$ in $2.5^{\circ}$ increments and for the trapezoid from $10^{\circ}$ to $20^{\circ}$. The Reynolds number is varied from 75,000 to 225,00 in 25,000 increments. Every colour map in Fig. 8 represents one end-plate configuration, and every tile is the described difference of overall sound pressure level between this end plate and the base configuration for the entire airfoil integration region. The range of the colour scheme is saturated at $\pm 10 \mathrm{~dB}$.
Overall, the three different end plates show a quite similar behaviour. For zero angle of attack, the end plates increase the self-noise sound pressure level for almost all flow speeds. For low angles of attack up to $12.5^{\circ}$, the sound pressure level difference fluctuates around $0 \mathrm{~dB}$, which results in a seemingly random pattern of decrease and increase in the overall sound pressure level. A possible explanation could be that for lower angles of attack the noise contribution from the tip becomes less important (Moreau et al. 2016). For lower angles of attack the pressure difference between suction and pressure side is reduced. This pressure difference drives the cross-flow around the tip which is the source of the tip vortex. Other studies on NACA 0012 airfoils have shown that the tip vortex strength is related to lift produced by the airfoil (Zaman et al. 2017) and that the tip vortex size and radius of curvature increase with angle of attack (Moreau and Doolan 2016). If the tip vortex strength is reduced, the sound radiation from interaction with the airfoil solid surface also decreases. Therefore, other source regions like the trailing edge could be dominant and not affected positively by the end plates. In addition, it is possible that aerodynamic noise is generated by flow over the trailing edge of the end plates themselves.

At higher angles of attack it can be seen that the end plates become more effective in reducing the noise at all flow speeds. The highest overall noise reduction achieved by the investigated end plates is $7 \mathrm{~dB}$ at $20^{\circ}$ and $R e=75,000$. The noise reduction does not seem to depend strongly on the shape of the end plates because all three configurations yield similar trends and values.

To further investigate the effect of the end plates, the power spectral densities at the reference point for the three
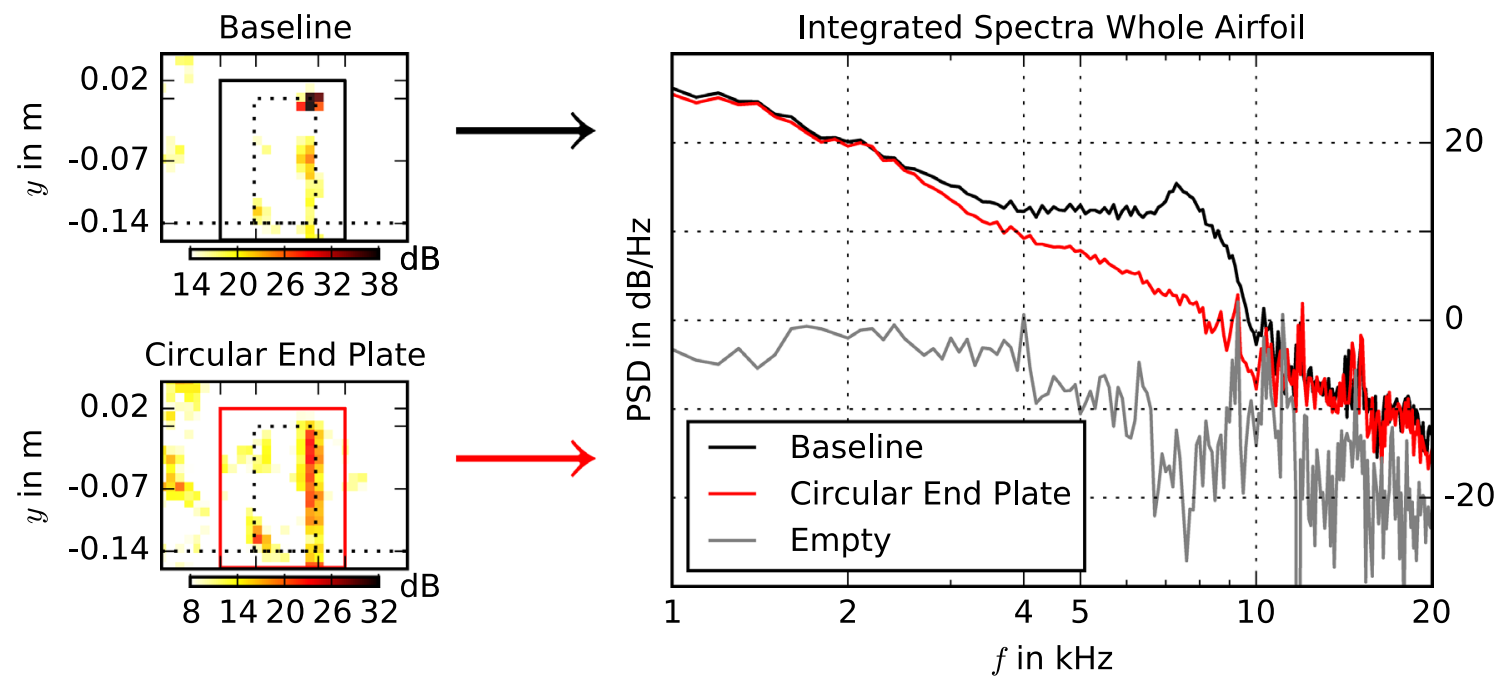

Fig. 7 Example for calculating overall sound pressure level differences of baseline and end plate: sources in sound maps on the left (examples for $6300 \mathrm{~Hz}$, airfoil black dashed line) are integrated

over sector (black, red) for every frequency band to obtain spectrum (right) and calculate OASPL difference for frequencies where the signal-to-noise ratio to the empty wind tunnel is more than $6 \mathrm{~dB}$ 

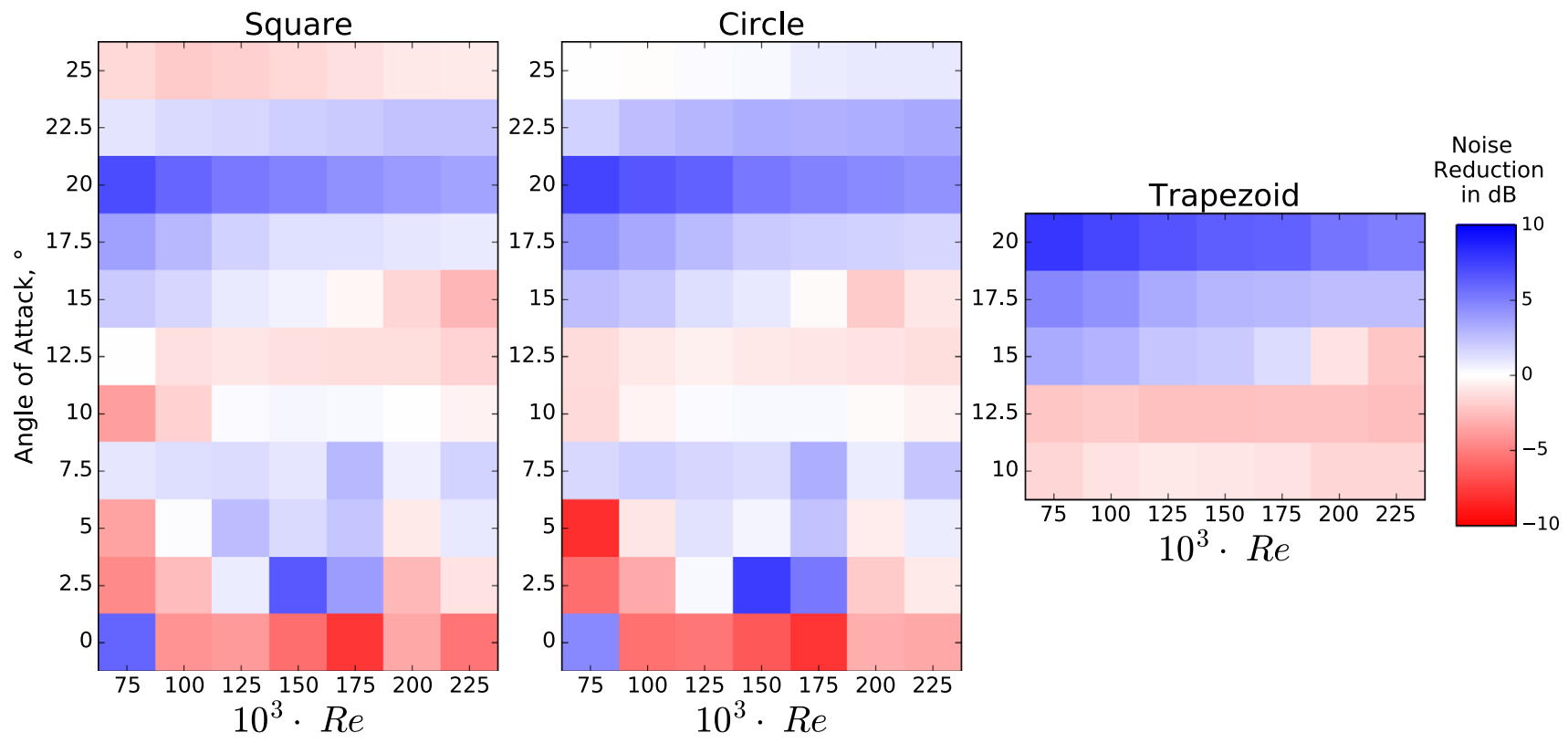

Fig. 8 Overall sound pressure level difference of integrated spectra for different tip configuration for frequency range 1 to $20 \mathrm{kHz}$ for NACA 0012 for different Reynolds numbers and angles of attack,

angles of attack with the highest noise reduction for all configurations and every second flow speed are plotted in Fig. 9. The Strouhal number and Reynolds number are based on the airfoil chord length. Results obtained for the lowest flow speed are shown as the spectra at the bottom, so that one row from left to right from Fig. 8 is shown here from bottom to top. The Strouhal number range shown is for the highest flow speed and frequency range from 1 to $20 \mathrm{kHz}$ where the signal-to-noise ratio is large enough.

The spectra for all angles of attack show a decrease in sound pressure level with increasing frequency except for the tripped baseline case at $10^{\circ}$. For $10^{\circ}$ and $15^{\circ}$ angle of attack, the baseline configuration shows a broadband hump that is located at Strouhal numbers between 18 and 20. This shows that the peak frequency is proportional to the flow velocity. With forced boundary layer transition, this peak is also apparent although the level decreases. This means that no laminar boundary layer instabilities are responsible for this hump.

Overall it can be seen that for all end-plate configurations this broadband peak vanishes and the decrease in sound pressure level continues with the same slope towards higher frequencies. The trapezoidal end plate develops a tonal peak that has a lower frequency than the broadband peak. Its Strouhal number is between 7 and 8 , and it most likely can be attributed to the vortex shedding from the sharp edges of the end plate in this configuration. The same applies to the square end plate at a slightly higher Strouhal number. For lower flow speeds, the sound pressure level of the integration area is whole airfoil, blue indicates a noise reduction and red a noise increase

high-frequency hump is higher compared to the broadband noise at low frequencies. For $15^{\circ}$ and $R e=75,000$, the level difference is around $5 \mathrm{~dB}$ while at $R e=225,000$ it is roughly $15 \mathrm{~dB}$. For $10^{\circ}$, the trend is similar except for the lowest flow speed where the hump is not existent. This trend explains why the noise reduction shown in Fig. 8 is more effective at low flow speeds.

For a geometric angle of attack of $20^{\circ}$, the spectral behaviour of the base configuration changes. The spectral hump is broadened compared to the other two angles of attack. While the lower frequency level and the slope of the spectra towards higher frequencies stay almost the same for the endplate configurations, the baseline spectra are a few $\mathrm{dB}$ higher in the complete frequency range. This leads to a high overall noise reduction, and once again the difference is larger for lower Reynolds numbers.

As an example for a fully turbulent case, the spectrum obtained for the tripped NACA 0012 airfoil with attached end plates is shown at $15^{\circ}$ angle of attack in Fig. 9. As observed for the untripped cases, the formation of the spectral hump is suppressed in the tripped cases by applying end plates as well. For the higher angles of attack where the noise reduction effect of the end plates is effective, the boundary layer transition seems to have no great influence on the far-field noise.

It is noticeable that all the shown sound pressure level spectra for the end-plate configurations have a similar shape compared to the far-field noise spectra from a different study on self-noise of airfoils in stall (Moreau et al. 2009). In that 

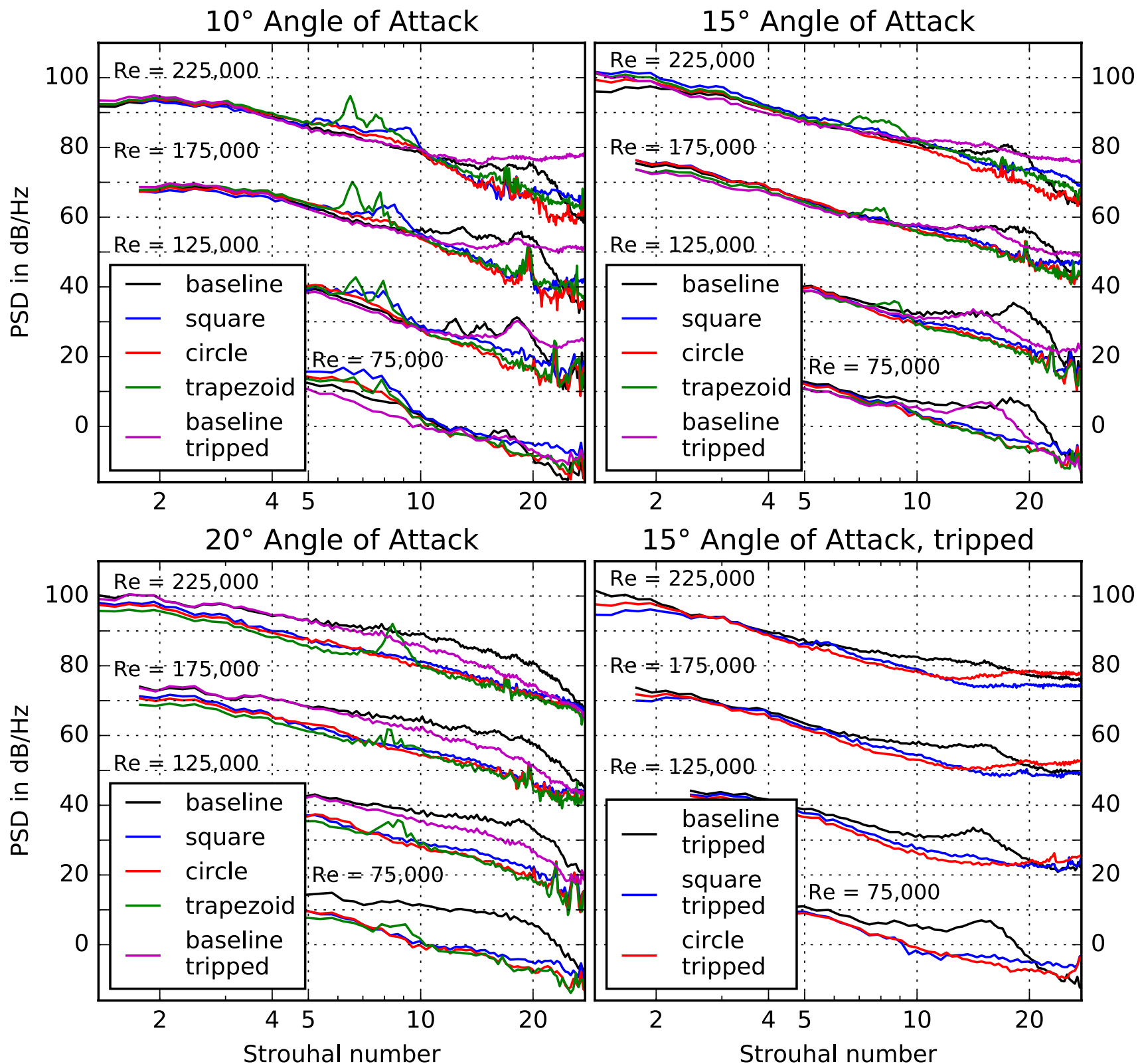

\section{$15^{\circ}$ Angle of Attack, tripped}

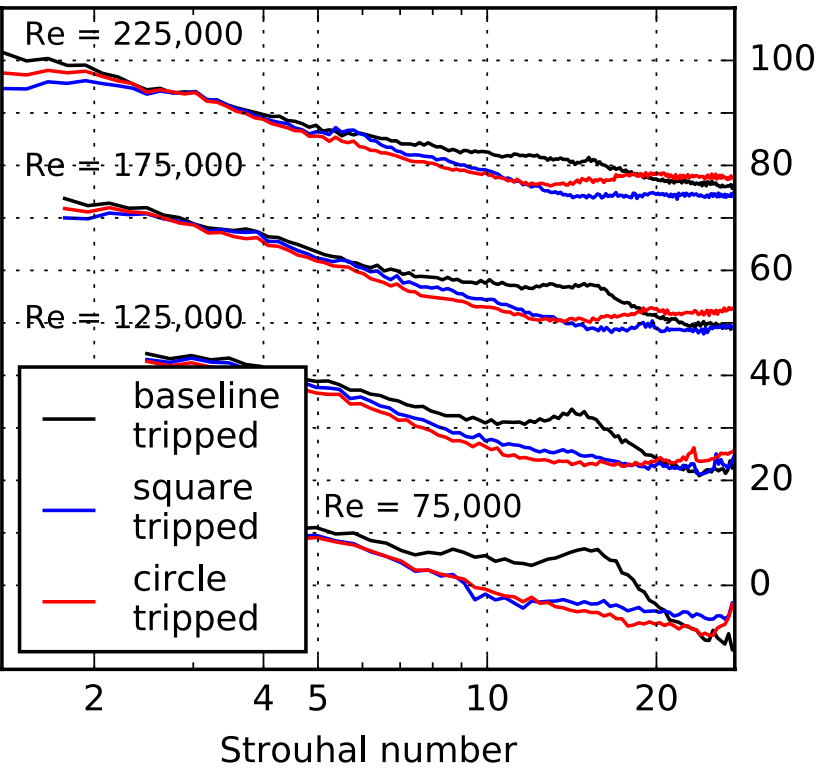

Fig. 9 Power spectral density for NACA 0012 airfoil for $10^{\circ}, 15^{\circ}, 20^{\circ}$ and $15^{\circ}$ angle of attack and every second flow speed for all end-plate configurations and the baseline case (tripped/untripped), the lowest

flow speed belongs to the bottom graphs, between each flow speed $20 \mathrm{~dB}$ are added to the spectra to avoid overlay (frequency resolution reduced to $100 \mathrm{~Hz}$ to increase visibility)

paper, NACA 0012 airfoils with aspect ratio $L / C 1.6$ were attached between two walls and the total pressure was measured close to the trailing edge to detect the airfoil flow condition. Except for the absolute level, the spectral shape of the far-field noise for stall conditions resembles that of the far-field noise observed in the current study for the endplate configurations. This indicates that in the current measurements stall occurred for higher angles of attack as well. The results for the end-plate configurations are close to the results of the 2D case from Moreau et al. (2009), where also no broadband frequency hump is apparent.
In the next step, the contribution of each integration area from Fig. 6 to the overall sound pressure level is investigated. Here, the circular end plate is chosen exemplarily, because it results in the highest noise reduction for most cases. Qualitatively, these results are similar to those of the other two configurations, which will not be shown here. In Fig. 10, the overall sound pressure level difference for the whole airfoil is shown and then the differences in SPL due to noise sources in the tip region, the leading edge (LE) region and the trailing edge (TE) region. Thereby, the contribution of noise reduction from a particular region needs to be 

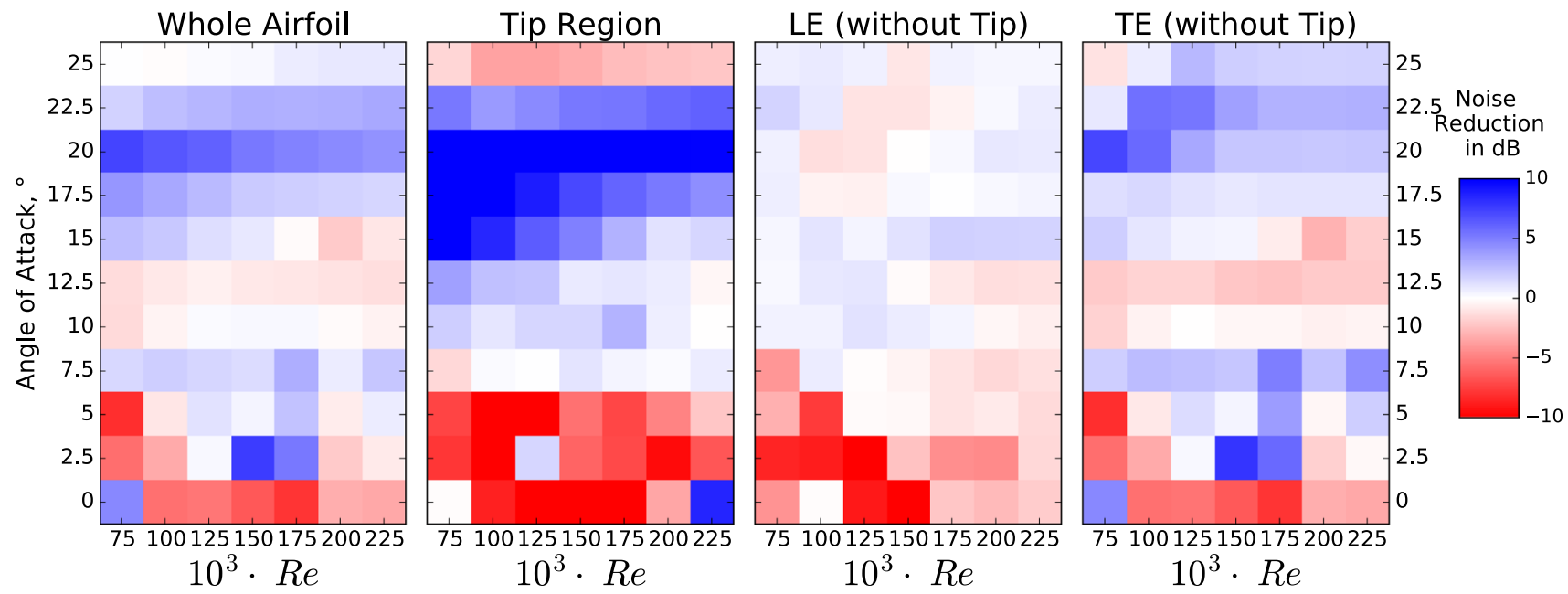

Fig. 10 Overall sound pressure level difference of integrated spectra for circular end plate for frequency range 1 to $20 \mathrm{kHz}$ for NACA 0012 for different Reynolds numbers and angles of attack, blue indicates a noise reduction and red a noise increase; for integration sectors see Fig. 6

considered in conjunction with the level difference of the whole airfoil; only if both show a noise reduction, this particular integration area is relevant. To improve distinctiveness of the different cases, the same colour bar as in Fig. 8 is used although the noise reduction reaches maximum values of up to $16 \mathrm{~dB}$ for some cases in the tip region.

For the configurations when the end plate is effective in decreasing noise, it can be seen that the reduction in the tip region is strongest. For these cases, a slight decrease in SPL at the leading and trailing edge can be observed. On the contrary, for lower angles of attack the SPL increases the strongest at the tip. The noise spectra for these cases show tonal peaks that get shifted to higher frequencies and higher amplitudes when the end plate is applied to the airfoil.

For a more detailed look at the noise source locations, third octave band sound maps for the dominant frequencies from the broadband hump are shown in Fig. 11 for the NACA 0012 base configuration and the circular end plate applied for $15^{\circ}$ angle of attack and $R e=125,000$. Although the difference of the overall sound pressure level is only $1.3 \mathrm{~dB}$ for this example, it still gives insight to important areas of noise generation. For all selected frequency bands,
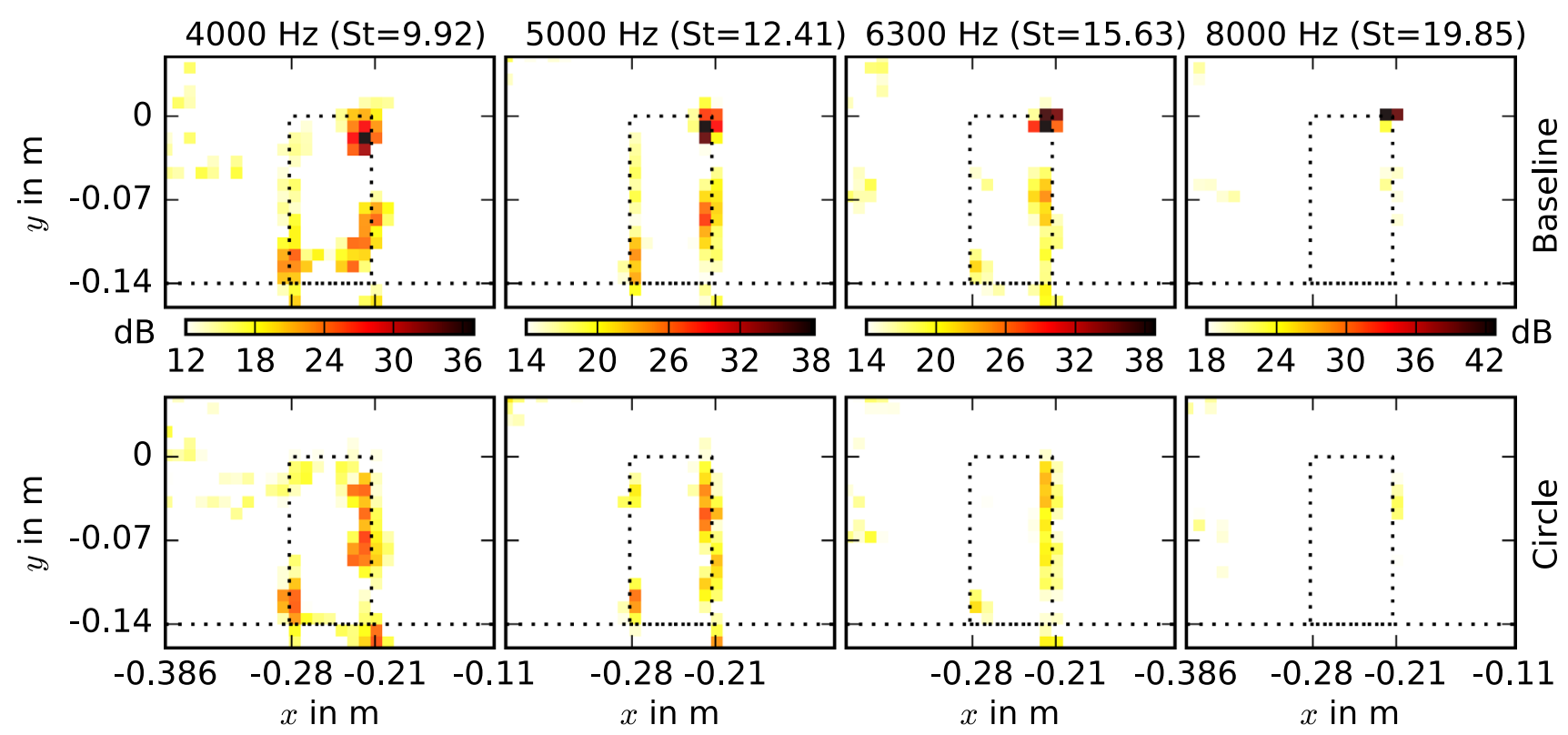

Fig. 11 NACA 0012 third octave band sound maps obtained at $15^{\circ}$ angle of attack for $R e=125,000$, top base configuration, bottom circular end plate, flow from left to right (nozzle exit at $-0.386 \mathrm{~m}$, black dotted line represents airfoil, grey dashed line represents wall) 
the base configuration shows a dominant sound source at the trailing edge of the wing tip. The sound pressure level of this source takes values that are between $10 \mathrm{~dB}$ and $18 \mathrm{~dB}$ higher than the strongest source observed for the circular end-plate configuration. Here, a large number of weaker noise sources are distributed over the whole span of the trailing edge as well as at the leading edge near the wall. This shows that for these frequency bands the most dominant sound source of the reference airfoil is at the tip and can be associated with tip vortex formation. By applying the circular end plate, this sound source is effectively suppressed.

\subsection{Beamforming results-NACA 4412}

In Fig. 12, the difference of the overall sound pressure level between the base configuration and the three end plates is shown for the NACA 4412 airfoil at the same Reynolds numbers and also additionally for $27.5^{\circ}$ and $30^{\circ}$ angle of attack. For this cambered airfoil, the trend is similar for all three end-plate shapes. For low Reynolds numbers and low angles of attack, an increase in sound pressure level can be observed. For angles of attack up to $12.5^{\circ}$ and higher Reynolds numbers, the trend rather reveals a noise reduction for the square and circular end plate, while the trapezoid shows a slight increase in sound pressure level. For the lower Reynolds number cases between $15^{\circ}$ and $20^{\circ}$, a higher reduction in sound pressure level can be observed than in the cases with higher flow speed. The maximum noise reduction is $10 \mathrm{~dB}$ for $20^{\circ}$ and $R e=100,000$. For these cases, the trapezoidal end plate shows a slight sound pressure level increase. A noise reduction can be seen for all flow speeds at angles of attack above $20^{\circ}$. This effect vanishes again above $25^{\circ}$ angle of attack. Compared to the NACA 0012 airfoil, a larger area of the measurement matrix shows favourable behaviour in reducing noise. In comparison with the symmetric NACA 0012 airfoil, no strong tonal peaks are apparent at low angles of attack. This may be due to the fact that flow separation is less likely to occur for the cambered airfoil due to the smaller surface pressure gradient (see Fig. 1).

The power spectral densities obtained for the NACA 4412 airfoil at four angles of attack are shown exemplary in Fig. 13 for every second flow velocity. For $10^{\circ}$ angle of attack, a broadband peak can again be observed in the upper frequency range. The highest sound pressure levels occur at a chord-based Strouhal number of around 12 for lower flow speeds and 15 for higher flow speeds. Again, a clear dependency of the peak frequency on the flow speed is seen, although in this case the Strouhal number is lower compared to that observed for the NACA 0012 airfoil. This peak is not apparent for all end-plate configurations. In comparison with the sound pressure level of the symmetric NACA 0012 at the same angle of attack, the center frequency of the peak
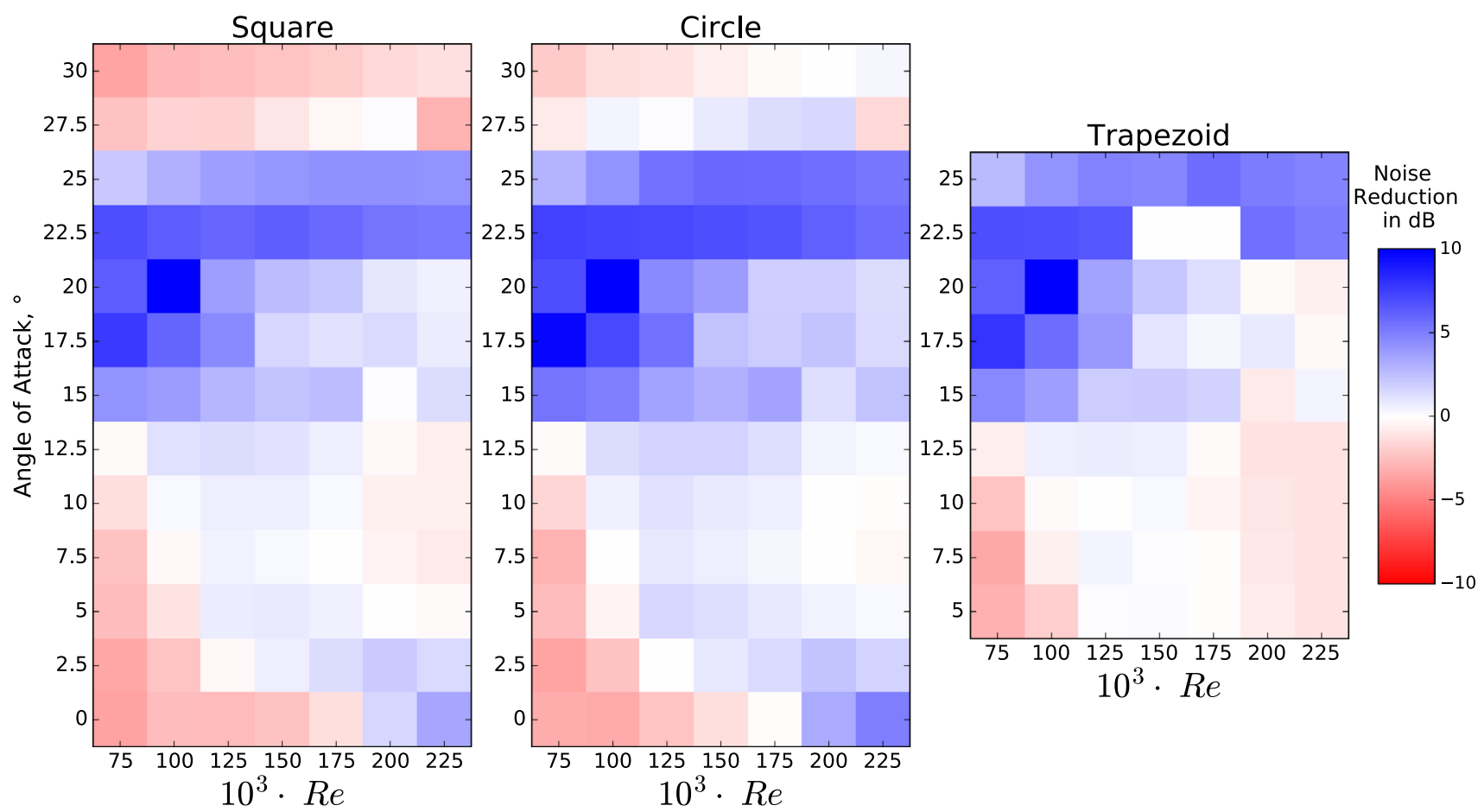

Fig. 12 Overall sound pressure level difference of integrated spectra for different tip configuration for frequency range 1 to $20 \mathrm{kHz}$ for integration area is whole airfoil, blue indicates a noise reduction and red a noise increase 

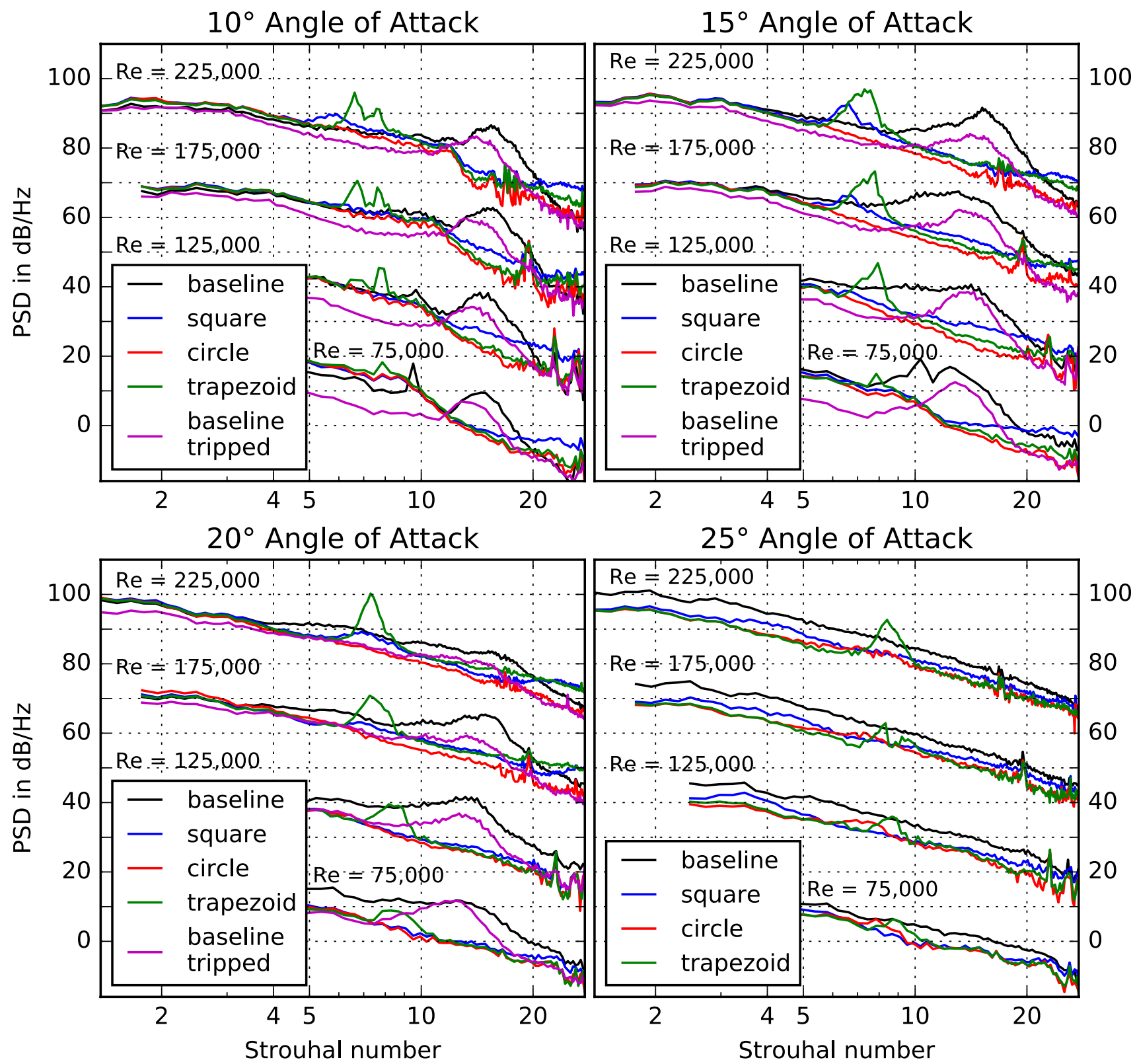

Fig. 13 Power spectral density for NACA 4412 airfoil for $10^{\circ}, 15^{\circ}$, $20^{\circ}$, and $25^{\circ}$ angle of attack and every second flow speed for all endplate configurations and the baseline case (tripped/untripped), the

lowest flow speed belongs to the bottom graphs, between each flow speed $20 \mathrm{~dB}$ are added to the spectra to avoid overlay (frequency resolution reduced to $100 \mathrm{~Hz}$ to increase visibility)

is lower for the NACA 4412 airfoil case. Also, the level of the peak does not decrease as strongly with increasing flow speed. For low flow speeds, it even exceeds the low frequency noise level. Therefore, the end plates are more effective in reducing the noise level than for the symmetric airfoil at the same angle of attack and Reynolds number. The tripped baseline configuration shows a less prominent hump, which is shifted towards lower frequencies and the mid-frequency levels are also reduced. As seen for the NACA 0012, it can be assumed that the end plates would also completely suppress the hump in the tripped configuration.

For the NACA 4412 at $15^{\circ}$ angle of attack not only the trapezoidal end plate but also the square plate develop a tonal peak for higher velocities that has a lower frequency than the broadband peak. It is assumed that vortex shedding from the sharp edges of these configurations causes this tonal noise contribution. In comparison with the sound pressure spectra obtained at $10^{\circ}$ angle of attack, the hump at $15^{\circ}$ is broadened, which increases the noise reduction effect of the end plates.

For $20^{\circ}$ angle of attack and the two highest flow speeds, the broadband peak of the baseline configuration is less 
prominent and has values below the level of the low-frequency broadband noise. Towards lower Reynolds numbers, the level of the peak rises significantly above the broadband level. The sound pressure level spectrum at the lowest Reynolds number shows a broadened peak that increases the sound pressure level by $6 \mathrm{~dB}$ down to $1 \mathrm{kHz}$ compared to the end-plate configurations. Once again the spectra of the end-plate configurations show a decrease in sound pressure level with a constant slope with increasing frequency and their amplitude therefore is below that of the baseline configuration. The only exception is the trapezoidal end plate that develops a tonal peak with increasing amplitude for increasing Reynolds number.

The far-field noise spectra observed at $25^{\circ}$ angle of attack show a different spectral behaviour. All four configurations result in the same spectral shape, but the spectrum obtained for the baseline configuration has a notably higher level than those of the end-plate configurations. The difference is in the order of $6 \mathrm{~dB}$ at lower frequencies and $3 \mathrm{~dB}$ at higher frequencies. This effect leads to a high overall noise reduction that is visible in Fig. 12 for this angle of attack.

As was done for the NACA 0012 airfoil, the noise contributions originating from different regions of the airfoil are investigated for the NACA 4412 airfoil as well, using the integration sectors from Fig. 6. The results are shown exemplary for the circular end plate in Fig. 14, because maximum noise reduction is achieved for this configuration. In the tip region, a high noise reduction is achieved for angles of attack between $12.5^{\circ}$ and $25^{\circ}$. The sound pressure level difference compared to the baseline configuration is above $10 \mathrm{~dB}$ for almost all operating conditions. Towards higher Reynolds numbers, the difference becomes smaller.
This effect is also observed in the spectra in Fig. 13, where with increasing flow velocity the level of the broadband peak becomes smaller in comparison with the rest of the spectra, and therefore, the noise reduction effect of the end plates decreases. For angles of attack below $12.5^{\circ}$, the difference decreases gradually. The $22.5^{\circ}$ and $25^{\circ}$ cases indicate that the trailing edge noise has a higher contribution to the overall sound pressure level because the high noise reduction at the tip does not affect the difference for the whole airfoil strongly. These are the cases where in Fig. 13 a different spectral behaviour is observed, and therefore, different flow mechanisms on the airfoil can be expected. For all other angles, no significant difference is observed except for the lowest Reynolds numbers where the level is increased.

The noise originating from the leading edge region is increased by a few $\mathrm{dB}$ for almost all cases from $0^{\circ}$ to $15^{\circ}$ angle of attack. For higher angles, the leading edge noise is not strongly affected by the circular end plate.

Selected third octave band sound maps are shown in Fig. 15 for the NACA 4412 airfoil at an angle of attack of $15^{\circ}$. The chosen frequencies belong to the broadband hump observed at a Reynolds number of 125,000. Results are compared for the baseline configuration and the circular end plate attached. Like the symmetric airfoil, the NACA 4412 baseline configuration shows the strongest sources at the trailing edge at the very tip. The noise reduction of the strongest source reaches values between $11 \mathrm{~dB}$ and $19 \mathrm{~dB}$ when the circular end plate is attached. The position of the sources is shifted a few centimeters down the span. In addition, other sources at the leading edge wall junction are apparent. Again, the end plate seems to suppress the strong sound source associated with the tip vortex and a
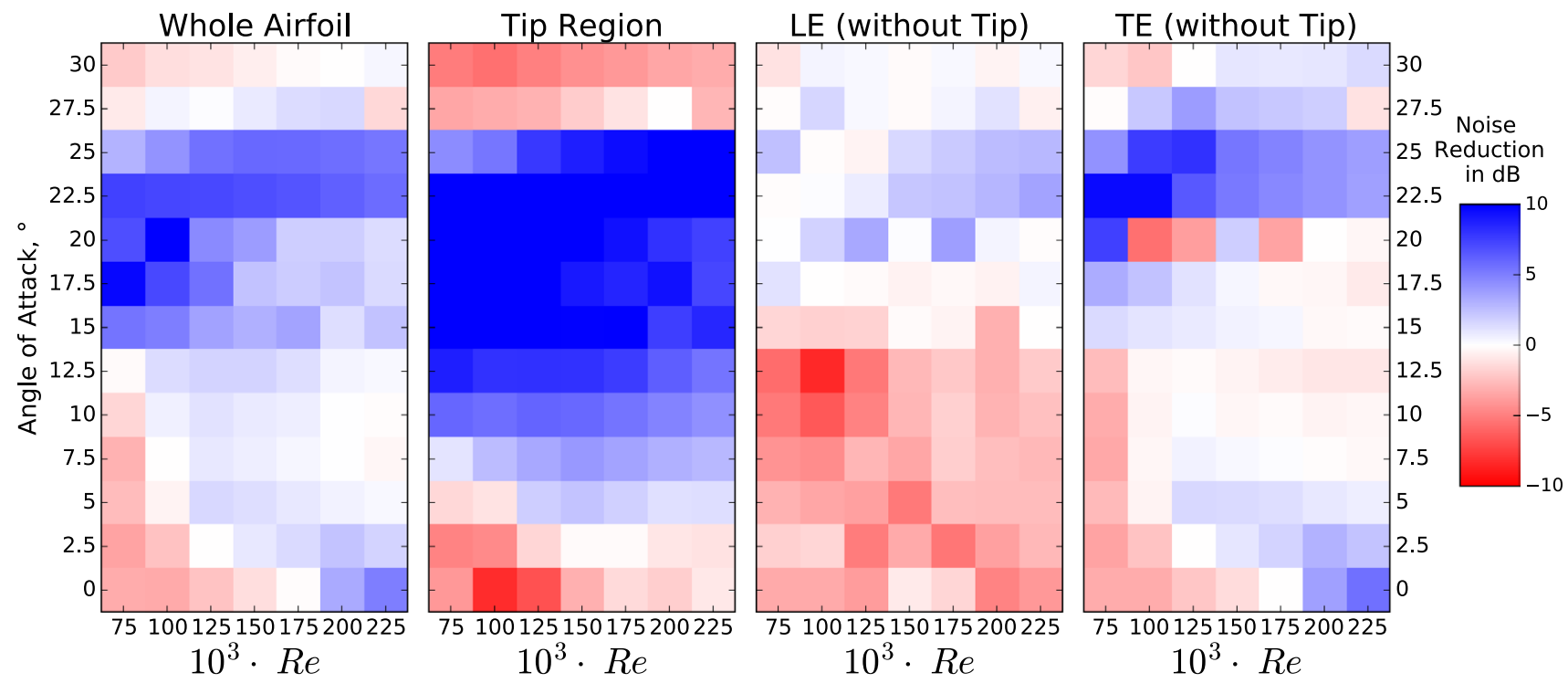

Fig. 14 Overall sound pressure level difference of integrated spectra for circular end plate for frequency range 1 to $20 \mathrm{kHz}$ for NACA 4412 for different Reynolds numbers and angles of attack, blue indicates a noise reduction and red a noise increase; for integration sectors see Fig. 6 

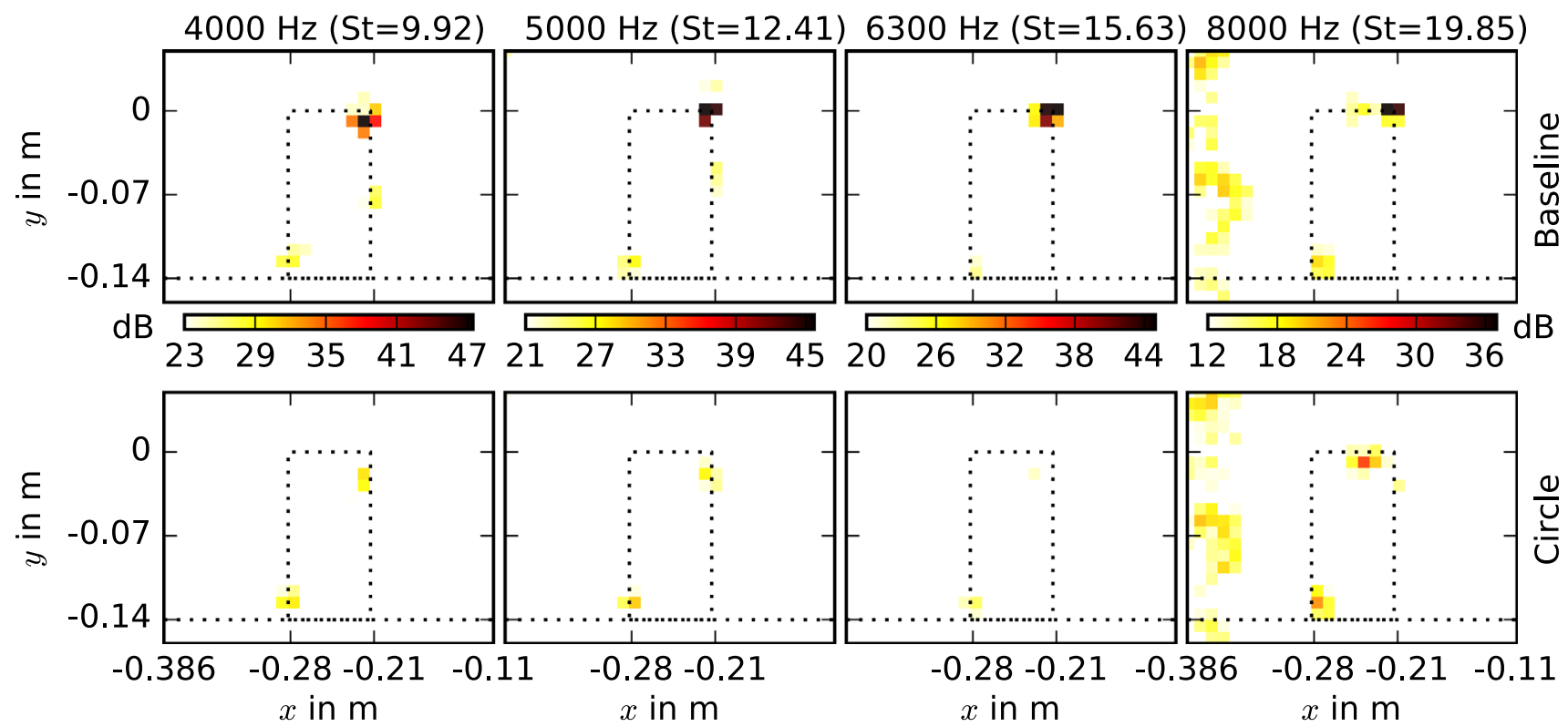

Fig. 15 NACA 4412 third octave band sound maps obtained at $15^{\circ}$ angle of attack for $R e=125,000$, top base configuration, bottom circular end plate (nozzle exit at $-0.386 \mathrm{~m}$, black dotted line represents airfoil, grey dashed line represents wall)

noise reduction of $3.6 \mathrm{~dB}$ is achieved for the overall sound pressure level.

\subsection{Hot-wire measurement results}

To improve understanding of the flow field at the airfoil tip, results from the hot-wire measurements are presented for the NACA 0012 at $15^{\circ}$ angle of attack and a Reynolds number of 125,000 . For this case, a broadband peak in the sound pressure level spectrum is apparent, and corresponding sound maps can be seen in Fig. 11. The first row in Fig. 16 shows the turbulence intensity (including the streamwise and vertical component) for the baseline configuration (left) and the configurations with circular (center) and rectangular (right) end plate. The measurement plane is located 0.1 chord lengths downstream of the airfoil trailing edge, the location of which is indicated in the plot. The airfoil ends at $(0,0)$ so that only the outermost $20 \mathrm{~mm}$ of the airfoil span are shown. The suction side is in the positive $z$-direction. Between the measurement points, the values are interpolated.

Two different flow fields can be observed: For the baseline case with no end plate applied the maximum turbulence intensity occurs along the trailing edge and around the tip in shape of a vortex structure that develops from the pressure side of the airfoil around the tip towards the suction side. The maximum turbulence intensity is around $16 \%$. The flow field in the case of both end-plate configurations shows notably different features. A high turbulence intensity can also be observed at the trailing edge, but additionally in a large spanwise area, elevated above the trailing edge, another high turbulence intensity region is present. The maximum intensity is significantly higher with $27 \%$ for the circular end plate and $24 \%$ for the rectangular end plate. A possible explanation for the different flow fields observed could be the free end, where the tip vortex is generated. For finite airfoils, the effective angle of attack decreases towards the tip (Brooks and Marcolini 1986; Awasthi et al. 2018), and the tip flow field has influence over the entire span (Moreau et al. 2018). This implicates that stall occurs at higher angles of attack compared to a two-dimensional airfoil, because new fluid from the pressure side can enter the boundary layer on the suction side and the flow remains attached to the airfoil surface for a longer downstream distance. This phenomenon was also observed by Genç et al. (2018), where for a NACA 4412 with $\mathrm{AR}=3$ the separation of the shear layer was suppressed by the formation of tip vortices, thus causing a delay of stall for angles of attack up to $40^{\circ}$ and Reynolds number 50,000. For higher Reynolds numbers, the finite airfoil acted as a 2D airfoil because the effect of viscous forces and leading edge vortices on the flow decreased. This could also apply to the study by Riley (1951), where a higher Reynolds number $\left(10^{6}\right)$ was investigated and where the influence of the tip was decreased due to the higher aspect ratio $(\mathrm{AR}=4)$. Therefore, delayed stall cannot be seen in Fig. 2a) where all configurations achieve maximum lift at the same angle of attack.

In contrast to the baseline case, it seems that for the endplate configurations the airfoil is in stall along the whole part of the span that is shown. The application of end plates seems to lead to more two-dimensional flow characteristics 

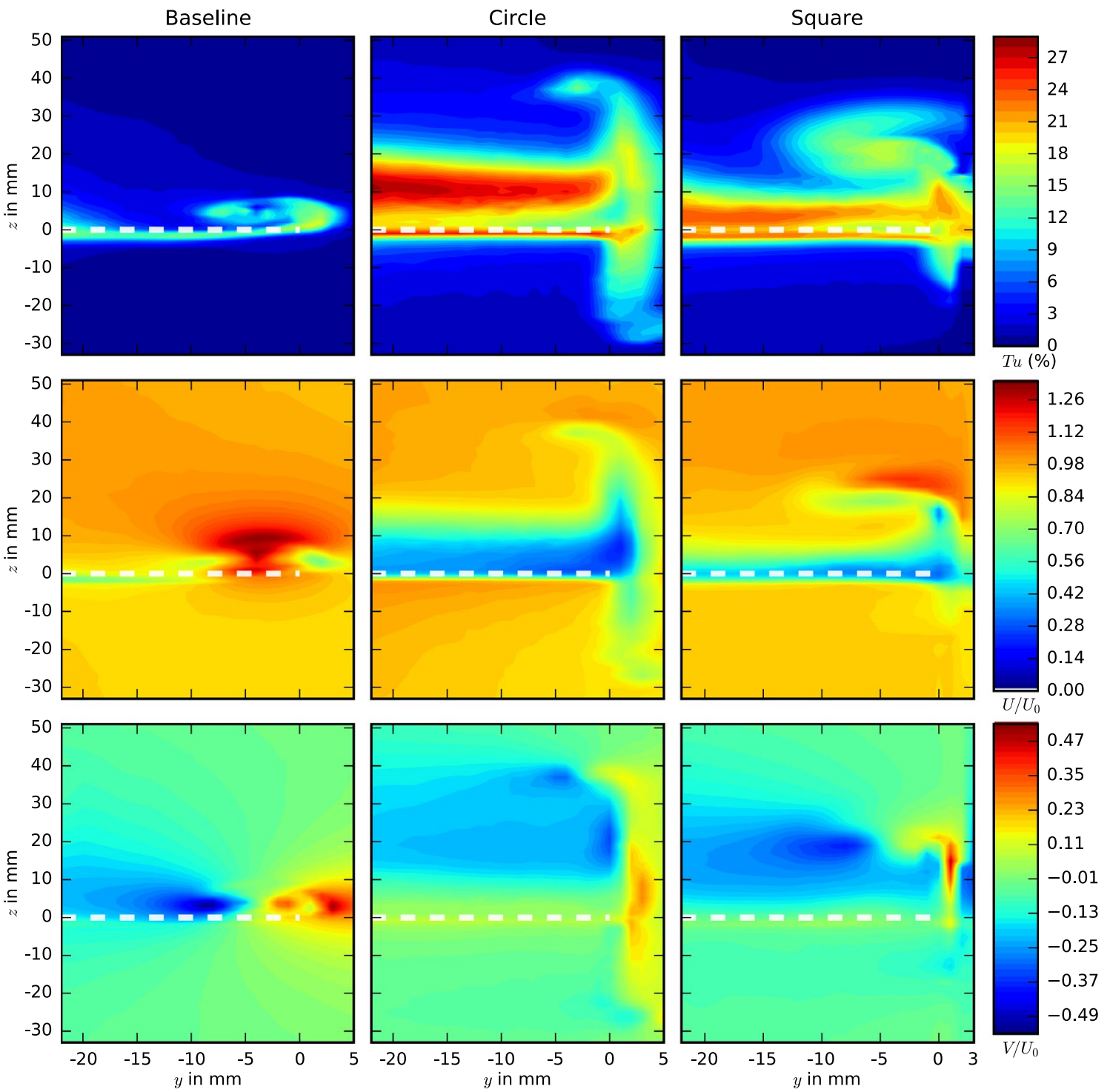

Fig. 16 Turbulence intensity, mean streamwise velocity $U$ and mean upwash velocity $W$ for NACA 0012 airfoil, angle of attack $15^{\circ}$, $R e=125,000$ [measurement plane located 0.1 chord lengths downstream of the airfoil, white dashed line represents the trailing edge,

at this angle of attack. The areas of high turbulence intensity above the trailing edge are assumed to be due to flow separation along the airfoil. The high turbulence intensity along the trailing edge could also be the reason for the acoustic source distribution that is seen in Fig. 11. Both end-plate cases also give reason to believe that some flow is still going around the top of the end plate. This effect is stronger for the smaller rectangular plate than for the circular end plate. The interaction of this swept flow with the flow over the airfoil seems to be small compared to the baseline configuration though, because the area of high turbulence intensity extends up to the end plate and is not affected. wall is located at $y=-140 \mathrm{~mm}$ and airfoil tip at $y=0 \mathrm{~mm}$, base configuration (left), circular end plate (center), rectangular end plate (right)]

The mean streamwise velocity is depicted in the second row of Fig. 16. The baseline configuration shows an area at the tip where the mean flow velocity is 1.3 times higher than in the undisturbed flow. The location and direction of this maximum confirm that it belongs to a vortex formed at the tip. For the two end-plate configurations, a notable area with low mean velocity is visible, which is stretched along the span and located just below the high turbulence intensity region observed in these cases. This is another indicator that stall occurs, as it shows the remaining flow over the trailing edge while most of the flow is separated due to stall. This area is again more pronounced for the larger circular end plate. 
Finally, the mean flow velocity in the vertical direction, perpendicular to the main flow and the trailing edge, is shown in the bottom row of Fig. 16. In the flow field on the very left, belonging to the baseline configuration, it can be seen that fluid from the pressure side of the airfoil is going upwards to the suction side around the airfoil tip (positive magnitude). Then, roughly $10 \mathrm{~mm}$ inwards down the span, the flow is going downwards again (negative magnitude). This indicates a tip vortex formation from pressure to suction side. Some upwards flow is also observed around the end plates as well as a roll-up around the top edge of the plates and the subsequent forming of a smaller vortex. Based on the absence of vortex induced noise in the acoustic results obtained for the end-plate configurations, it appears, though, that this vortex does not interact with the airfoil trailing edge due to the increased vertical distance.

The results from the hot-wire measurements clearly show the absence of an airfoil tip vortex for the end-plate configurations. In the sound maps of Fig. 11, no strong sound sources were found at the tip for these configurations, and the broadband frequency hump is not apparent in the spectra. This confirms the tip noise reduction effect of the end plates.

\section{Conclusions}

This paper presents the experimental investigation of the noise reduction effect of three different end-plate designs on symmetric and cambered wall-mounted finite airfoils. The measurements were taken in a small aeroacoustic wind tunnel using microphone array technique and advanced beamforming algorithms that enable the determination of both the location and the strength of the noise sources. In addition, constant temperature anemometry measurements were taken on selected configurations to allow for a better understanding of the flow structures present.

As a first result, beamforming sound maps revealed that for the baseline configurations at high geometric angles of attack a dominant sound source is located at the trailing edge of the tip. These high-frequency broadband sound sources are greatly reduced when the end plates are applied. Corresponding hot-wire measurements downstream of the tip region showed a significant change of the flow field when the end plates are applied. The results suggest that the end plates greatly reduce the transfer of fluid from the pressure to the suction side and, subsequently, diffuse the vorticity at the tip. Thus, the influence of flow structures forming at the free end of the airfoil is reduced and the flow field rather resembles that of a two-dimensional configuration.

Corresponding sound pressure level spectra obtained from the microphone array measurements further confirm that the noise at the tip region can be reduced by applying end plates to the free end of the airfoil. For the cases without end plates, the spectra feature a notable broadband highfrequency hump associated with the tip vortex formation. This hump is not apparent for the end-plate configurations. Since the hump is more pronounced in the spectra for the asymmetric NACA 4412 airfoil, the overall noise reduction was found to be greater for this airfoil than for the symmetric NACA 0012 airfoil. The achieved noise reduction at the tip is highest for higher angles of attack for both airfoils. For the NACA 4412 noise reduction was achieved for almost all Reynolds numbers at geometric angles of attack from $2.5^{\circ}$ to $25^{\circ}$ except for the lower $R e$. The end plates on the NACA 0012 airfoil were effective in reducing noise at geometric angles of attack from $15^{\circ}$ to $22.5^{\circ}$. The results showed that the overall sound pressure level can only be reduced if the tip region is the dominant source region. Otherwise the sound pressure levels are not affected or sometimes even slightly increased by the presence of the end plates. For very high angles of attack, the end plates lead to a noise reduction over the whole frequency range.

The exact shape of the end plate did not have a strong effect on the acoustic results although the circular end plate, which has the largest area, showed the best results in noise reduction. This dependency as well as the effects of end plates on the flow over the airfoil surface and the resulting aerodynamics is of interest for future research.

Acknowledgements Partial funding for this project was provided by the German Academic Exchange Service (DAAD) under the Project-ID 57445107, which is gratefully acknowledged. The authors also thank Tingyi Zhang and Charitha de Silva for their help with the flow visualization experiments.

Funding Open Access funding enabled and organized by Projekt DEAL.

Open Access This article is licensed under a Creative Commons Attribution 4.0 International License, which permits use, sharing, adaptation, distribution and reproduction in any medium or format, as long as you give appropriate credit to the original author(s) and the source, provide a link to the Creative Commons licence, and indicate if changes were made. The images or other third party material in this article are included in the article's Creative Commons licence, unless indicated otherwise in a credit line to the material. If material is not included in the article's Creative Commons licence and your intended use is not permitted by statutory regulation or exceeds the permitted use, you will need to obtain permission directly from the copyright holder. To view a copy of this licence, visit http://creativecommons.org/licenses/by/4.0/.

\section{References}

Albertson ML, Dai YB, Jensen RA, Rouse H (1950) Diffusion of submerged jets. Trans Am Soc Civil Eng 115(1):639-664

Angland D, Zhang X, Molin N (2009) Measurements of flow around a flap side edge with porous edge treatment. AIAA J 47:1660-1671

Awasthi M, Moreau D, Doolan C (2018) Flow structure of a low aspect ratio wall-mounted airfoil operating in a low Reynolds number flow. Exp Therm Fluid Sci 99:94-116 
Bahr CJ, Humphreys WM, Ernst D, Ahlefeldt T, Spehr C, Pereira A, Leclère Q, Picard C, Porteous R, Moreau D, et al (2017) A comparison of microphone phased array methods applied to the study of airframe noise in wind tunnel testing. In: 23rd AIAA/CEAS aeroacoustics conference, AIAA paper 2017-3718

Brooks TF, Marcolini MA (1986) Airfoil tip vortex formation noise. AIAA J 24:246-252

Doolan CJ, Moreau DJ, Awasthi M, Jiang C (2018) The UNSW anechoic wind tunnel. In: Proceedings of acoustics vol 7

Drela M (1989) Xfoil: an analysis and design system for low reynolds number airfoils. In: Low Reynolds number aerodynamics. Springer, pp. 1-12

Genç MS, Özkan G, Özden M, Kiriş MS, Yildiz R (2018) Interaction of tip vortex and laminar separation bubble over wings with different aspect ratios under low Reynolds numbers. Proc Inst Mech Eng Part C J Mech Eng Sci 232:4019-4037

George AR, Najjar FE, Kim YN (1980) Noise due to tip vortex formation on lifting rotors. AIAA Paper

Geyer TF, Sarradj E, Herold G (2015) Flow noise generation of cylinders with soft porous cover. In: 21st AIAA/CEAS aeroacoustics conference, AIAA paper 2015-3147

Guo Y (2011) Aircraft flap side edge noise modeling and prediction. In: 17th AIAA/CEAS aeroacoustics conference 2011 (32nd AIAA aeroacoustics conference) $5-8$

Hald J (2017) Removal of incoherent noise from an averaged crossspectral matrix. J Acoust Soc Am 142:846-854

Hardin JC (1980) Noise radiation from the side edges of flaps. AIAA J 18:549-552

Herold G, Sarradj E (2017) Performance analysis of microphone array methods. J Sound Vib 401:152-168

Kinzie K, Drobietz R, Petitjean B, Honhoff S (2013) AWEA Windpower 2013 Chicago , IL May 6-8 , 2013 Concepts for wind turbine sound mitigation

Merino-Martínez R, Sijtsma P, Snellen M, Ahlefeldt T, Antoni J, Bahr CJ, Blacodon D, Ernst D, Finez A, Funke S et al (2019) A review of acoustic imaging methods using phased microphone arrays. CEAS Aeronaut J 10:197-230

Moreau DJ, Doolan CJ (2016) An experimental study of airfoil tip vortex formation noise. In: Proceedings of acoustics 2:1167-1176

Moreau S, Roger M, Christophe J (2009) Flow features and self-noise of airfoils near stall or in stall. In: 15th AIAA/CEAS aeroacoustics conference (30th AIAA aeroacoustics conference) pp 11-13

Moreau DJ, Doolan CJ, Alexander WN, Meyers TW, Devenport WJ (2016) Wall-mounted finite airfoil-noise production and prediction. AIAA J 54:1637-1651
Moreau DJ, Geyer TF, Doolan CJ, Sarradj E (2017) Camber effects on the tonal noise and flow characteristics of a wall-mounted finite airfoil. In: 23rd AIAA/CEAS aeroacoustics conference, AIAA paper 2017-3172

Moreau DJ, Geyer TF, Doolan CJ, Sarradj E (2018) Surface curvature effects on the tonal noise of a wall-mounted finite airfoil. J Acoust Soc Am 143:3460-3473

Revell JD, Kuntz HL, Balena FJ, Home C, Storms BL, Dougherty RP (1997) Traeling-edge flap noise reduction by porous acoustic treatment. In: 3rd AIAA/CEAS aeroacoustics conference pp 493-505

Riley DR (1951) Wind-tunnel investigation and analysis of the effects of end plates of the aerodynamic characteristics of an unswept wing. Technical Report

Sarradj E, Herold G (2017) A Python framework for microphone array data processing. Appl Acoust 116:50-58

Sarradj E, Fritzsche C, Geyer TF, Giesler J (2009) Acoustic and aerodynamic design and characterization of a small-scale aeroacoustic wind tunnel. Appl Acoust 70:1073-1080

Sarradj E, Herold G, Sijtsma P, Merino Martinez R, Geyer TF, Bahr CJ, Porteous R, Moreau D, Doolan CJ (2017) A microphone array method benchmarking exercise using synthesized input data. In: 23rd AIAA/CEAS aeroacoustics conference, AIAA paper 2017-3719

Sijtsma P (2007) Executive summary CLEAN based on spatial source coherence. Int J Aeroacoust 6:357-374

Slooff JW, de Wolf WB, van der Wal HM, Maseland JE (2002) Aerodynamic and aero-acoustic effects of flap tip fences. In: 40th AIAA aerospace sciences meeting and exhibit pp 1-29

Zaman KBMQ, Fagan AF, Mankbadi MR (2017) An experimental study and database for tip vortex flow from an airfoil. NASA Technical Memorandum

Zhang W, Cheng W, Gao W, Qamar A, Samtaney R (2015) Geometrical effects on the airfoil flow separation and transition. Comput Fluids 116:60-73

Zhang T, Moreau D, Geyer TF, Fischer J, Doolan C (2020) Dataset on tip vortex formation noise produced by wall-mounted finite airfoils with flat and rounded tip geometries. Data in Brief 28:105058

Publisher's Note Springer Nature remains neutral with regard to jurisdictional claims in published maps and institutional affiliations.

\section{Authors and Affiliations}

\section{Erik Schneehagen ${ }^{1} \cdot$ Thomas F. Geyer $^{2} \cdot$ Ennes Sarradj $^{1} \cdot$ Danielle J. Moreau ${ }^{3}$}

\author{
Thomas F. Geyer \\ thomas.geyer@b-tu.de \\ Ennes Sarradj \\ ennes.sarradj@tu-berlin.de \\ Danielle J. Moreau \\ d.moreau@unsw.edu.au
}

1 Technische Universität Berlin, Einsteinufer 25, 10587 Berlin, Germany

2 Brandenburg University of Technology Cottbus-Senftenberg, Siemens-Halske-Ring 15A, 03046 Cottbus, Germany

3 University of New South Wales, Sydney, NSW 2052, Australia 